\title{
Wissen und Umwelt in der „partizipatorischen Diktatur"
}

\author{
Wissenschaftliche Umweltkonzepte und der umweltpolitische \\ Aufbruch in der DDR
}

\author{
Christian Möller
} Knowledge and Environment in the "Participatory Dictatorship". Scientific Environmental Concepts and the
Environmental Policy Change in the GDR

The GDR was an ecological failed state in 1989/90. But while public input and critical environmental groups protested more openly against environmental problems during the 1980s, the established sciences remained strangely mute and appeared unable to develop appropriate approaches to solving the environmental crisis. Almost 20 years earlier, however, an environmental policy departure that was largely supported by scientific reform initiatives had begun in the GDR. The inclusion of the concept of conserving nature and the environment in the 1968 constitution and the adoption of the land improvement law two years later were the expression of a socio-political consensus on the environmental issue.

How can this sharp discrepancy be explained? The article investigates the influence of scientific environmental concepts on the departure in environmental policy in the GDR. The example of scientific nature conservation, which had been institutionally anchored in the German Academy of Agricultural Sciences since 1951, shows that conservationists underwent a period of "normalization" in the 1950s, which was an essential prerequisite for giving their concerns political legitimacy. This forced adaptation process not only influenced the concepts and goals of East German nature conservation, rather also opened up participatory opportunities for its protagonists.

The essay argues for a reassessment of East German environmental history, which so far has only been considered from the perspective of how it ended. Furthermore, it is argued that the conceptual concept of the "participatory dictatorship" (Fulbrook) should be applied to the history of science in the GDR in order to expand existing approaches.

Keywords: Nature conservation, Socialist land improvement, German Academy of Agricultural Sciences, "participatory dictatorship", Research council, Waste products

1989/90 war die DDR ein ökologisch gescheiterter Staat. Doch während Eingaben aus der Bevölkerung und kritische Umweltgruppen im Laufe der 1980er Jahre immer offener gegen die vorhandenen Umweltprobleme protestierten, blieben die etablierten Wissenschaften merkwürdig stumm und waren allem Anschein nach nicht in der Lage, geeignete Konzepte zur Lösung der Umweltkrise zu entwickeln. Knapp 20 Jahre zuvor hatte jedoch in der DDR ein umweltpolitischer Aufbruch eingesetzt, der maßgeblich von wissenschaftlichen Reforminitiativen getragen wurde. Die Aufnahme des Natur- und Umweltschutzgedankens in die Verfassung von 1968 und die Verabschiedung des Landeskulturgesetzes zwei Jahre darauf, waren Ausdruck eines gesellschaftspolitischen Konsenses in der Umweltfrage.

Wie ist diese scharfe Diskrepanz zu erklären? Der Aufsatz fragt nach dem Einfluss wissenschaftlicher Umweltkonzepte auf den umweltpolitischen Aufbruch in der DDR. Am Beispiel des wissenschaftlichen Naturschutzes, der seit 1951 institutionell in der Deutschen Akademie der Landwirtschaftswissenschaften verankert war, wird gezeigt, dass Naturschützer in den 1950er Jahren eine Phase der „Normalisierung" durchliefen, die eine wesentliche Voraussetzung dafür war, ihren Anliegen politische Legitimation zu verleihen. Dieser erzwungene Anpassungsprozess beeinflusste aber nicht nur die Konzepte und Ziele des ostdeutschen Naturschutzes, sondern eröffnete seinen Protagonisten auch Partizipationsmöglichkeiten. 
In diesem Aufsatz plädiere ich für eine Neubewertung der ostdeutschen Umweltgeschichte, die bislang einseitig vom Ende her blickt. Zudem schlage ich vor, das begriffliche Konzept der "partizipatorischen Diktatur" (Fulbrook) auf die Wissenschaftsgeschichte der DDR zu übertragen und vorhandene Ansätze damit zu erweitern.

Schlüsselwörter: Naturschutz, Sozialistische Landeskultur, Deutsche Akademie der Landwirtschaftswissenschaften, „partizipatorische Diktatur", Forschungsrat, Abprodukte

Die Ökobilanz der DDR fiel bitter aus (Petschow et al. 1990). Dennoch scheinen die zahlreichen ökologischen Verfehlungen des SED-Staates, mit denen auch massive Repressionen gegen politisch Andersdenkende einhergingen, vor der politischen Wende im Herbst 1989 kaum auf Kritik gestoßen zu sein. Folgt man den Befunden des noch zu DDR-Zeiten eingerichteten "Zentralen Runden Tisches“ oder der wenige Jahre darauf vom Deutschen Bundestag einberufenen Enquete-Kommission „Aufarbeitung von Geschichte und Folgen der SED-Diktatur", wagten es lediglich wenige hundert Umweltaktivisten, darunter auch Wissenschaftler des Kirchlichen Forschungsheims in Wittenberg, unter dem Schutzdach der evangelischen Kirchen offene Kritik an vorhandenen Problemen zu üben (Bechmann 1991: 87-89; Schlußbericht 1998: 11-117, 326, 332 f.). Während das Umweltengagement dieser Akteure auf vielfache Weise behindert wurde, scheinen sich die zuständigen Staatsorgane und gesellschaftlichen Organisationen darüber ausgeschwiegen, Umweltprobleme negiert und sich ansonsten lediglich in ideologischer Phraseologie geübt zu haben. Auch die Wissenschaftler, von denen die westlichen Umweltbewegungen in den Jahrzehnten zuvor nachweislich wichtige Impulse erhalten hatten (Hünemörder 2004: 275-296; Radkau 2011: 116-123, 169-173), hielten sich allem Anschein nach mit öffentlicher Kritik zurück. Prominente Kritiker aus den eigenen Reihen, die die Wachstumsideologie des Marxismus-Leninismus offen in Frage stellten und von der Führungsspitze ein umweltpolitisches Umdenken forderten, wurden hingegen mundtot gemacht. ${ }^{1}$

Blickt man von der politischen Wende 1989/90 jedoch 20 Jahre zurück, zeigt sich ein ganz anderes Bild: Im Jahr 1968 wurde der Natur- und Umweltschutz als Staatsziel in Artikel 15 der neuen Verfassung aufgenommen (Verfassung 1968: 16). Zwei Jahre darauf verabschiedete die Volkskammer mit dem Landeskulturgesetz eines der europaweit ersten Rahmengesetze für den Schutz der Umwelt. ${ }^{2}$ Im Jahr 1971 veranlasste der Ministerrat schließlich den Aufbau eines Ministeriums für Umweltschutz und Wasserwirtschaft, das gut 14 Jahre vor seinem westdeutschen Pendant die Arbeit aufnahm. ${ }^{3}$ Zwischen 1970 und 1973 diskutierten Funktionäre von SED, Blockparteien, Massenorganisationen und volkseigenen Betrieben während sogenannter "Wochen der sozialistischen Landeskultur" landesweit 
die Umweltsituation. Der Kulturbund und die Nationale Front mobilisierten in den darauffolgenden Jahren zehntausende Bürgerinnen und Bürger, die sich in staatlich sanktionierten Initiativen für den Natur- und Umweltschutz engagierten. (Möller 2018: 244-258, 349-351) Auch die Akademie der Wissenschaften der DDR folgte diesem Trend und richtete zu Beginn der 1970er Jahre eine Klasse „Umweltschutz und Umweltgestaltung“ sowie eine Forschungsstelle für Umweltgestaltung ein (Paucke 1994: 113, 119; Kocka et al. 2002: 382). Umweltschutz war, so scheint es zumindest, zu einem politischen Konsensthema geworden.

Wie ist diese binnen nur weniger Jahre entstandene, scharfe Diskrepanz zu erklären? Im vorliegenden Aufsatz wird dieser Frage nachgegangen und am Beispiel des ostdeutschen Naturschutzes der Einfluss wissenschaftlichen Umwelthandelns sowie wissenschaftlicher Umweltkonzepte auf einen umweltpolitischen Aufbruch untersucht, der offenkundig in der zweiten Hälfte der 1960er Jahre in der DDR eingesetzt hatte. Ferner wird danach gefragt, wie Umweltwissen durch wissenschaftliche Disziplinen konzipiert wurde. Wie gelang es wissenschaftlichen Akteuren, die für eine Umsetzung ihrer Anliegen notwendige politische Legitimation von der SED-Führung $\mathrm{zu}$ erhalten? Und wie veränderte diese Interaktion zwischen Wissenschaft und Politik umgekehrt das Selbstverständnis der beteiligten akademischen Disziplinen? Dazu werden im Folgenden Veröffentlichungen führender Naturschützer sowie Archivmaterial aus dem Umfeld der Deutschen Akademie der Landwirtschaftswissenschaften ausgewertet. Die vorliegende Untersuchung beruht ferner auf Teilergebnissen meiner Dissertation, die an dieser Stelle insbesondere in konzeptionell-theoretischer Hinsicht vertieft werden (Möller 2018).

Bevor die oben genannten Fragen erörtert werden können, gilt es in einem ersten Schritt zunächst das Verhältnis von Politik und Wissenschaft in der sozialistischen Diktatur näher zu bestimmen. Denn anders, als in den freiheitlich-demokratisch verfassten Gesellschaften des Westens, konnte eine kritische Umweltdebatte in der DDR nur innerhalb der engen Grenzen der sozialistischen Herrschaftsordnung entstehen. Die durch die Machtmechanismen der Diktatur strukturell bedingte Verborgenheit gesellschaftlicher Aushandlungsprozesse führte dazu, so die Ausgangsthese meiner Argumentation, dass sich nach der Wiedervereinigung ein „master narrative“ etablieren konnte, das den ökologischen Niedergang der DDR als unausweichliche Entwicklung darstellte und in jüngster Zeit immer stärker in Kritik geraten ist (Roesler 2006: 480 f.; Obertreis 2012: 115, 118; Möller 2018: 17-20). Die katastrophale Umweltbilanz stützte allem Anschein nach die von totalitarismustheoretisch beeinflussten Studien und ehemaligen Oppositionellen vertretene Annahme, wonach die massiven Umweltprobleme in der DDR durch Konstruktionsfehler der Planwirtschaft und 
ein sämtliche Umweltbelange ignorierendes Primat der Ökonomie „systemisch vorherbestimmt" gewesen wären. Legale Kritik an den vorhandenen Umweltproblemen oder gar konformer Umweltprotest waren dieser Lesart zufolge schon aufgrund der diktatorischen Strukturen nicht möglich gewesen. Kritisches Umweltengagement hätte sich immer der Gefahr einer Verfolgung durch den Staat ausgesetzt. Der umweltpolitische Aufbruch der 1960er und frühen 1970er Jahre verkümmerte infolgedessen in der öffentlichen und historischen Wahrnehmung zu einer Alibistrategie des Politbüros, die im Vorfeld der ersten Umweltkonferenz der Vereinten Nationen im Jahr 1972 in Stockholm einzig das Ziel verfolgte, die Überlegenheit des Sozialismus gegenüber dem Kapitalismus zu demonstrieren und eine diplomatische Anerkennung der DDR durch die internationale Staatengemeinschaft zu erreichen. ${ }^{4}$

Dieses teleologische Geschichtsbild soll im Folgenden am Beispiel des Umwelthandelns von naturpolitisch engagierten Wissenschaftlern innerhalb der Deutschen Akademie der Landwirtschaftswissenschaften (DAL) kritisch hinterfragt werden. Anhand der Karriere des agrarischen Landeskulturbegriffes, der von Vertretern der Akademie zu einem spezifisch ostdeutschen Vorläufermodell des modernen Umweltbegriffes weiterentwickelt wurde, werde ich darlegen, dass der wissenschaftliche Naturschutz in den 1950er und 1960er Jahren einen Prozess der inneren und äußeren Anpassung durchlief. Diese Phase der „Normalisierung“ (Fulbrook) war eine grundlegende Voraussetzung für den Erfolg des umweltpolitischen Aufbruchs, der sich in der Verabschiedung des Landeskulturgesetzes im Jahr 1970 ausdrückte. Wissenschaftler wie Hans Stubbe, Hermann Meusel, Ludwig Bauer und Hugo Weinitschke wirkten auf eine moderate Anpassung des Naturschutzes an die Bedürfnisse einer modernen Industriegesellschaft hin und erhielten daraufhin Zugang zu Expertennetzwerken, die ab 1967 die konzeptionellen Grundlagen der sozialistischen Umweltpolitik erarbeiteten. Diese Einbindung des Naturschutzes und die dahinterstehenden Aushandlungsprozesse gründeten auf vorhandenen autoritärkorporatistischen Partizipationsmöglichkeiten, die zwar restriktiv ausgelegt, in diesem Sinn aber konstitutiv für die SED-Herrschaft waren und somit auch die Handlungsbedingungen für ein naturpolitisches Engagement ostdeutscher Wissenschaftler prägten. Andere Faktoren des umweltpolitischen Aufbruchs, wie beispielsweise Eingabenproteste aus der Bevölkerung, die in den 1960er Jahren zunahmen und nachweislich ebenfalls einen großen Einfluss auf die Formierung der sozialistischen Umweltpolitik ausübten, können an dieser Stelle hingegen nicht berücksichtigt werden (Möller 2018: 173-185). 


\section{Die Polyvalenz der Ressourcenmobilisierung oder: Wissenschaft in der „partizipatorischen Diktatur"}

Die Beziehungsgeschichte von Wissenschaft und Staat war auf dem Gebiet der SBZ/DDR nach 1945 durch zwei Pole geprägt: Zum einen stellte sich - wie in anderen modernen Industriegesellschaften auch - ein wechselseitiges Abhängigkeitsverhältnis zwischen diesen beiden Sphären ein, das an Entwicklungen anknüpfte, die bereits in der ersten Hälfte des 20. Jahrhunderts eingesetzt hatten, nun allerdings ein qualitativ neues Niveau erreichten. Während der gesellschaftliche Teilbereich „Wissenschaft" infolge neuer politischer und sozialer Anforderungen einen Prozess der Ökonomisierung durchlief und in diesem Sinne nützliches Wissen für Volkswirtschaft, Volksgesundheit und andere Gesellschaftsbereiche produzieren sollte, nahmen umgekehrt die Einflussmöglichkeiten wissenschaftlicher Experten auf die Politik zu. Die kontinuierlich anwachsende Komplexität technischer und ökonomischer Sachverhalte machte auch die sozialistische Staatsführung immer abhängiger von wissenschaftlichen Expertisen, ohne dass dabei allerdings jemals das Primat der Politik ernsthaft infrage gestellt war. Dieser Trend wurde zum anderen dadurch verschärft, dass ostdeutsche Wissenschaftler in einem ideologisch und politisch besonders aufgeladenen Kontext interagierten. Denn die SED begriff Wissenschaft nicht nur als ein Instrument zur Steigerung der wirtschaftlichen Leistungsfähigkeit oder zur Verbesserung der Arbeits- und Lebensbedingungen der Bevölkerung, sondern bezog aus diesem Feld auch in besonderem Maße politische Legitimation. (Kocka 1998: 438 f.; Laitko 2009: 8 f.) In den 1960er Jahren, als die ideologischen Formeln von der „Produktivkraft Wissenschaft" und einer zu forcierenden "wissenschaftlich-technischen Revolution“ (WTR) auch im öffentlichen Raum der Diktatur immer präsenter wurden, war die Verquickung des politischen Machtanspruches der SED mit der Kategorie des wissenschaftlichen Fortschritts allenthalben spürbar (Kocka 1998: 455; Sabrow 2004: 178-180; Wolle 2011: 154-156).

Dennoch verfügte die SED in den unmittelbaren Nachkriegsjahren zunächst über keine einheitliche Strategie in der Wissenschaftspolitik. Steuerungsversuche beschränkten sich in den späten 1940er und 1950er Jahren vornehmlich darauf, die Macht der mehrheitlich noch bürgerlich geprägten Ordinarien zu brechen und durch einen wissenschaftlichen Nachwuchs zu ersetzen, der den ideologischen Ansprüchen der Partei genügen sollte. Widerstände aus den Natur-, Technik- und Gesundheitswissenschaften, die sich ihres hohen politischen Stellenwertes bewusst waren, bis 1961 vorhandene Ausweichmöglichkeiten nach Westdeutschland und ein nicht zuletzt daraus resultierendes Zaudern der politischen Führung bei der Umsetzung der anvisierten personellen "Säuberungen“ verhinderten bis in die 1960er 
Jahre hinein immer wieder, dass dieses Ziel konsequent umgesetzt werden konnte. Wie Ralph Jessen mit Blick auf das akademische Milieu dieser Jahrzehnte resümierend festhält, scheint die wissenschaftspolitische Strategie der SED auf personeller Ebene eher einem widersprüchlichen „Nacheinander von Eliteverdrängung, Eliteintegration und Eliteaustausch mit erheblichen fachspezifischen Unterschieden“ (Jessen 1998: 51) als jenem ideologisch hochstilisierten „Sturm auf die Festung Wissenschaft" geglichen zu haben, der in den ersten Nachkriegsjahren immer wieder vollmundig von Parteifunktionären gefordert wurde (Kowalczuk 1997: 104, 115).

Auf institutioneller Ebene gelang es der SED hingegen sehr viel früher bedeutende Schritte hin zu einem sozialistischen Umbau der Wissenschaftslandschaft anzustoßen. Bereits im Sommer 1946 hatte die Sowjetische Militäradministration in Deutschland der Wiedereröffnung der Preußischen Akademie der Wissenschaften (PAW) zugestimmt, die nun in Deutsche Akademie der Wissenschaften zu Berlin (DAW) umbenannt wurde. Die DAW erfuhr in den folgenden Jahren einige entscheidende strukturelle Veränderungen, die sowohl an das Vorbild der sowjetischen Akademie der Wissenschaften anknüpften als auch älteren Reformbemühungen aus den Reihen der preußischen Vorgängereinrichtung folgten. Die wichtigste strukturelle Neuerung stellte der Aufbau von akademieeigenen Forschungsinstituten dar, wodurch sich die DAW sukzessive von einer Gelehrtengesellschaft zu einer Forschungsakademie wandelte. Die DAW trat infolgedessen zunehmend in Konkurrenz zu den etablierten Universitäten und übte mittels der wissenschaftlichen Expertise, die sich in ihren Forschungsinstituten bündelte, großen Einfluss auf politische Entscheidungen aus. $^{5}$

Die Machtposition der DAW wurde allerdings durch eine Reihe weiterer institutioneller Umgestaltungen und Neugründungen eingeschränkt. $\mathrm{Zu}$ Beginn der 1950er Jahre entstanden mit der Bauakademie (1950) und der Deutschen Akademie der Landwirtschaftswissenschaften (DAL, 1951) zwei bedeutende Teilakademien, die ebenfalls mit Forschungsinstituten ausgestattet wurden und in den 1980er Jahren - gemessen an ihrer Mitarbeiterstärke - fast 20 beziehungsweise mehr als 45 Prozent der DAWForschungskapazitäten erreichten (Kocka et al. 2002: 423). Des Weiteren entstand im Jahr 1957 mit dem Forschungsrat eine weitere einflussreiche wissenschaftliche Institution, die auf den Feldern der Wissenschaftspolitik und der Politikberatung in Konkurrenz zur Akademie trat. Das offiziell als „Beirat für naturwissenschaftlich-technische Forschung und Entwicklung" betitelte Gremium war direkt beim Ministerrat angesiedelt und übte Einfluss auf die Aufstellung von Wissenschaftsplänen sowie die Verteilung von Forschungsgeldern aus, begutachtete überdies wirtschaftliche Planungen und beteiligte sich an der Vorbereitung von Perspektivplänen. 
Im Gegensatz zur DAW verfügte das Gremium jedoch über keine eigenen Forschungseinrichtungen. Die Besonderheit des Forschungsrates, dessen Plenum sich in den 1960er Jahren aus weniger als 50 handverlesenen Wissenschaftlern zusammensetzte, bestand in der engen Zusammenarbeit mit den Staats- und Wirtschaftsorganen. In Zentralen Arbeitskreisen und zeitlich befristet oder auch dauerhaft eingerichteten Kommissionen erarbeiteten führende Wissenschaftler zusammen mit Staats- und Wirtschaftsfunktionären langfristige Gesellschaftsprognosen, legten Lösungskonzepte für konkrete technische, wirtschaftliche oder gesellschaftliche Problemfelder vor und formulierten Beschlussvorlagen sowie Gesetzesentwürfe für die Führungsgremien von Partei und Staat. ${ }^{6}$

Die Staats- und Parteiführung weitete ihren wissenschaftspolitischen Kontroll- und Steuerungsanspruch indes nach dem Bau der Mauer 1961 massiv aus: Mit der bereits kurz zuvor erfolgten Aufwertung des Zentralamtes für Forschung und Technik in ein Staatssekretariat und der Gründung eines Ministeriums für Wissenschaft und Technik im Jahr 1967 wurden die bis dahin noch relativ großen Mitsprachemöglichkeiten der etablierten wissenschaftlichen Institutionen immer weiter beschnitten. Die Auflösung der Fakultäten und Institute zugunsten größerer Organisationseinheiten im Zuge der ebenfalls 1967 eingeleiteten „3. Hochschulreform“ machte den letzten Bastionen der bürgerlich geprägten Wissenschaft ein Ende. Durch die wiederum ein Jahr darauf begonnene „Akademiereform“ wandelte sich die DAW zudem endgültig in ein außeruniversitäres „Forschungskombinat".7 Parallel zu diesen vornehmlich wissenschaftsbezogenen Entwicklungen setzte auch innerhalb der parteieigenen Verwaltungsstrukturen ein Prozess der „Professionalisierung und Verwissenschaftlichung" ein, der den Einfluss externer wissenschaftlicher Expertisen spürbar hemmte (Kaiser 1997: 37-47; Bergien 2017: 254-259).

Die wissenschaftspolitischen Eingriffe der 1950er und 1960er Jahre, mit denen eine massive Ausdehnung des Kontrollanspruches der SED über die vorhandenen wissenschaftlichen Institutionen und das Wissenschaftspersonal verbunden waren, prägen bis heute das Bild einer ideologisierten und ökonomisierten Wissenschaftslandschaft, die sich von einem Hort des bürgerlichen Beharrungsvermögens zu einem profanen Funktionsbereich der SED-Diktatur wandelte. Wissenschaftlerinnen und Wissenschaftler wurden in diesem Zusammenhang häufig als „willfährige“ Konformisten dargestellt, die einstmals vorhandene Handlungsspielräume freiwillig opferten und sich der Partei bereitwillig als fachliche Herrschaftsressourcen zur Verfügung stellten. Die Konformität, die das wissenschaftliche Personal in der DDR - wie auch schon zuvor im Nationalsozialismus - an den Tag legte, erscheint in diesen Darstellungen häufig in einem negativen Wortsinn als Kollaboration, bestenfalls aber als Ausdruck von Ohnmacht. Die 
erdrückende Herrschaftsmaschinerie der totalitären SED-Diktatur mit ihrem allumfassenden Kontrollanspruch führte demnach einen „Prozeß des Absterbens der Gesellschaft" herbei, der auch das Feld der Wissenschaft erfasste. $^{8}$

Gegen diese Sichtweise regte sich allerdings Widerspruch: Der Wissenschaftshistoriker Mitchell G. Ash stellte 1995 die These auf, dass Wandel in der deutschen Wissenschaftsgeschichte des 20. Jahrhunderts vornehmlich auf die "Umgestaltung von Ressourcenkonstellationen“ zurückzuführen gewesen sei, die sich infolge politischer Machtwechsel und den daraus hervorgehenden neuen "Verwicklungen“ von Wissenschaftlern und Herrschenden einstellte. Unter Ressourcen versteht Ash dabei in Anlehnung an Bruno Latour und Andrew Pickering finanzielle, institutionelle, technische, kognitiv-konzeptionelle und rhetorische Mittel, die sowohl aus der wissenschaftlichen als auch der politischen Sphäre stammen konnten. Das mit Blick auf ein gewandeltes Verständnis der ostdeutschen Wissenschaftsgeschichte Besondere an diesem Ansatz ist die Annahme, dass diese Ressourcen grundsätzlich polyvalent mobilisierbar waren. Das Wissenschaftspersonal und wissenschaftliche Institutionen erscheinen bei Ash nicht mehr per se als passive Akteure in einer entdifferenzierten und abgestorbenen Gesellschaft, als Opfer von Regimewechseln und totalitären Diktaturen oder kurzum als Spielbälle übergeordneter politischer Entwicklungen, sondern vielmehr als Teilnehmer sozialer und politischer Interaktionen, die durchaus dazu in der Lage waren, aktiv Einfluss auf die Gestaltung ihrer eigenen Ressourcenkonstellationen zu nehmen. (Ash 1995: 3 f.; Ash 2001: 118-120, 130-132; Ash 2002: 32-34). Neuere Studien dehnen diesen Ansatz auch auf die Beziehungsebene zu weiteren Sphären, beispielsweise das Verhältnis von Wissenschaft und Öffentlichkeit, aus (Nikolow \& Schirrmacher 2007: 26 f.).

Auch von anderer Seite wurden schlichte wissenschaftshistorische Topdown-Modelle infrage gestellt. So betonte Jürgen Kocka, dass von Fach zu Fach unterschiedlich stark ausgeprägte Funktionsimperative dazu beigetragen hätten, ein gewisses Maß an wissenschaftlicher Selbststeuerung über die gesamte Dauer der DDR zu bewahren. Innere Wirkmechanismen hätten die Wissenschaften schon aus fachspezifischen Selbsterhaltungsgründen zur Grenzziehung gegenüber den Durchdringungsversuchen der SED gezwungen. Wissenschaft und Politik gingen Kocka zufolge demgegenüber vielmehr eine begrenzt symbiotische Beziehung ein, die es Wissenschaftlern nicht nur erlaubte Ressourcen zu mobilisieren, sondern umgekehrt auch Bereiche ihrer fachlichen Arbeit vor dem Einfluss der Partei abzuschirmen und von der Nähe zum Regime zu profitieren. Doch während die eine Seite dieser Beziehungsgeschichte, die Wissenschaftspolitik der ostdeutschen Partei- und Staatsführung, von der Forschung bereits um- 
fangreich untersucht worden sei, monierte Kocka, dass die andere Seite - die Gestaltungsmöglichkeiten von Wissenschaftlern und der politische Einfluss ihrer Expertisen - demgegenüber stark unterbelichtet erscheine (Kocka 1998: 445 f., 454-456).

Der von Ash formulierte Ansatz einer polyvalenten Ressourcenmobilisierung kann jedoch nur bedingt dabei helfen, diese Lücke zu schließen, da er bezogen auf sein Untersuchungsfeld zu selbstreferentiell bleibt. Das Handeln von Wissenschaftlern wird hier einseitig in Hinblick auf die Mobilisierung materieller und ideeller Ressourcen für die Aufrechterhaltung beziehungsweise die Verbesserung der eigenen Forschungsbedingungen betrachtet, wohingegen ein über die eigene wissenschaftliche Sphäre hinausreichendes Handeln, beispielsweise in Form eines gesellschaftspolitischen Engagements, nicht berücksichtigt wird. Wie das Beispiel des Naturschutzes zeigen wird, war aber genau diese Handlungsebene entscheidend für den umweltpolitischen Aufbruch in der DDR. Die Frage nach gesellschaftspolitischen Mitgestaltungsmöglichkeiten in der sozialistischen Diktatur berührt eine Debatte, die nach 1990 in der Gesellschaftsgeschichte geführt wurde. Das Wiedererstarken von totalitarismustheoretischen Ansätzen nach der Wiedervereinigung rief in der sich neuformierenden historischen DDR-Forschung schnell Widerspruch hervor. Zahlreiche Studien betonten demgegenüber die "Grenzen der Diktatur" und forderten dazu auf, Herrschaft auch im ostdeutschen Fall als eine soziale Praxis zu begreifen (Bessel \& Jessen 1996; Lindenberger 1999). Dennoch blieben diese Arbeiten im Hinblick auf die Interpretation und die Einordnung der von ihnen identifizierten Freiräume zurückhaltend. So wurde immer wieder betont, dass die Grenzen, die der Diktatur auf vielfältige Weise auferlegt waren, umgekehrt natürlich umso mehr für die Beherrschten galten. Die erkämpften Freiheiten blieben aber auch in diesen Arbeiten letztlich auf gesellschaftliche Nischen und eigen-sinnige Verhaltensformen beschränkt. Die vorhandenen "Räume des Mitmachens in der SED-Diktatur" sind bis heute kaum erforscht, wie Thomas Lindenberger unlängst feststellte (Lindenberger 2016).

Jenseits der unverrückbaren Eckpfeiler der Macht, die die Partei- und Staatsführung fraglos mit harter Hand verteidigte, war das Verhältnis zwischen Herrschenden und Beherrschten aber eher durch profane und wenig bedrohliche sowie zähe und kontroverse Aushandlungsprozesse als durch totalitäre Gewalt geprägt. Martin Sabrow verweist aus kulturhistorischer Perspektive auf die „konsensstiftenden Denkstrukturen und Wahrnehmungsmuster[n]“, die die Wirklichkeit in der sozialistischen Gesellschaft beeinflussten und für die Herrschaft der SED konstitutiv waren. Nicht Repression, sondern das Einverständnis der Beherrschten sei die schärfste Waffe der Diktatur gewesen und erkläre, warum das ostdeutsche 
Staatsgebilde über 40 Jahre hinweg so erstaunlich stabil war (Sabrow 1999: 90 f.). Sabrow bezeichnet die DDR daher als „Konsensdiktatur“, die von einer Ambivalenz aus konstitutivem „Konsenszwang“ und konstituierendem „Zwangskonsens“ geprägt war, in der die Grenzen von Diktat und Diskussion „bis zur Ununterscheidbarkeit" verwischt wurden (Sabrow 2001: 421, 446 f.). Dieses „Konsensprinzip“ eröffnete demnach auch die Möglichkeit zu offenem Dissens, so lange sich dieser innerhalb der dominanten Diskursordnung bewegte, während Dissens außerhalb der Diskursgrenzen hingegen harte Reaktionen nach sich zog. Dies hatte Auswirkungen auf alle am Diskurs beteiligten Akteure - auch auf die Wissenschaften, wie Sabrow am Beispiel der ostdeutschen Historiographie nachweist. (Sabrow 2001: 397).

Während Sabrow in der Konsensdiktatur in erster Linie eine diskursive Formation ausmacht, die prägend für die politische Kultur der DDR war, geht die Sozialhistorikerin Mary Fulbrook einen Schritt weiter und nimmt die Handlungsebene in den Blick. Sie betont, dass viele Ostdeutsche direkt oder indirekt mit dem SED-Staat verwickelt waren - sei es durch die Mitgliedschaft in der SED, einer Blockpartei, einer gesellschaftlichen Organisation oder die politische Kommunikation in Eingaben - und in diesem Sinn durchaus Einfluss auf politische Entscheidungen und die Ausgestaltung des gesellschaftlichen Zusammenlebens ausüben konnten. Fulbrook verwendet daher den Begriff der „partizipatorischen Diktatur“, der veranschaulichen soll, „dass die Menschen durch das sich ständig verändernde soziale und politische System der DDR eingeschränkt und beeinflusst wurden, es gleichzeitig aber auch aktiv und oft freiwillig trugen (Fulbrook 2008: 28)." Die Regeln für diese begrenzte Form der Teilhabe am gesellschaftlichen und politischen Leben im SED-Staat hatten die meisten Menschen spätestens in den 1960er Jahren internalisiert oder für die Durchsetzung ihrer Interessen zu nutzen gelernt. In diesem Jahrzehnt identifiziert Fulbrook eine Periode der „Normalisierung“, die vom Bau der Mauer, einem generationellen Übergang sowie einer daraus hervorgehenden Verlässlichkeit von Strukturen und Institutionen getragen wurde (Fulbrook 2008: 24, 213; Fulbrook 2009: 1-30; Fulbrook 2016: 71-76).

Der Begriff der „partizipatorischen Diktatur“ ist - wie hier gezeigt werden soll - auch dazu geeignet, den Ressourcenmobilisierungsansatz nach Ash entscheidend zu erweitern und die Wissenschaftsgeschichte der DDR in einen breiteren gesellschaftshistorischen Kontext einzuordnen. Ebenso, wie andere Bereiche der ostdeutschen Gesellschaft, lässt sich auch das $\mathrm{Ne}$ beneinander von wissenschaftspolitischen Steuerungsversuchen und wissenschaftlichem Beharrungsvermögen in den ersten beiden Nachkriegsjahrzehnten als eine Form der „Normalisierung“ verstehen. Dieser sowohl von diktatorischer Gewalt als auch von Konsens geprägte Prozess zog aller- 
dings nicht, wie oft angenommen, ein Verstummen der wissenschaftlichen Akteure nach sich. Wie das Beispiel des wissenschaftlichen Naturschutzes zeigen soll, eröffnete erst die Konformität der nach Teilhabe strebenden Akteure jene Handlungsmöglichkeiten, die die Formierung und Etablierung einer sozialistischen Umweltpolitik in der DDR in den 1960er Jahren möglich machten.

\section{Konformität als Schlüssel zur Partizipation: Der Naturschutz in der Phase der „Normalisierung” und der Konsenscharakter des Landeskulturbegriffs}

Umweltprobleme erregten in der DDR - wie in anderen Industrieländern auch - nicht erst in den 1970er Jahren gesellschaftliche und politische Aufmerksamkeit. ${ }^{9}$ Schon in den Jahrzehnten zuvor wurden einzelne Problembereiche, wie etwa vorhandene Störungen des Landschaftsbildes oder die Zunahme von Schadstoffbelastungen in den Gewässern und der Luft, thematisiert und zum Gegenstand wissenschaftlicher und technisch-administrativer Initiativen gemacht. ${ }^{10}$ Diese für den umweltpolitischen Aufbruch späterer Jahre konstitutive Phase war jedoch eine Zeit der vorwiegend verdeckt geführten Umweltdebatten. Die Partei- und Staatsführung betrachtete die vorhandenen Umweltbelastungen bis dahin, ähnlich wie auch die politischen Führungen westlicher Industriestaaten, in erster Linie als unvermeidbare Begleiterscheinung der industriellen Produktionsweise, die es auf technische und administrative Weise lokal zu beheben oder einzudämmen galt. Eine neue, umfassende politische Agenda, die diese Probleme mit einem ganzheitlichen Ansatz zu lösen versuchte, lag hingegen weder im Interesse der politischen Führung noch war sie Gegenstand der vorhandenen Reforminitiativen (Möller 2018: 54 f.).

Die SED bekam den zunehmenden Unmut der Bevölkerung über die Umweltprobleme an der Basis allerdings immer häufiger zu spüren und sah sich daher zu punktuellen Maßnahmen veranlasst. Eine erste Zäsur stellte der "Neue Kurs“ dar, den die Parteiführung unmittelbar im Vorfeld des Volksaufstandes vom 17. Juni 1953 verkündete. Die SED schenkte nun nicht nur den von Teilen der Arbeiterschaft als zu hoch empfundenen Arbeitsnormen und unerfüllten Konsumbedürfnissen der Bevölkerung größere Aufmerksamkeit, sondern befasste sich auch mit den Arbeits- und Lebensbedingungen der Menschen. ${ }^{11}$ Der Aufstand leitete zudem das Ende der rigiden Reparationspolitik ein, die die UdSSR zunächst eingeschlagen hatte. Mit der Rückgabe der letzten Betriebe, die nach 1945 von den Besatzern beschlagnahmt und in Sowjetische Aktiengesellschaften umgewan- 
delt worden waren, befanden sich alle großen „Umweltsünder“ wieder in ostdeutschem Staatsbesitz (Karlsch 1993: 129-135). Die SED trug nun unmittelbar die Verantwortung für Konflikte, die aus den industriellen Emissionen dieser Großbetriebe hervorgingen. Das sich in den Jahren darauf auch in der DDR einstellende Wirtschaftswachstum verschärfte die vielerorts bereits angespannte Lage. Die Partei- und Staatsorgane sahen sich mit Konflikten aufgrund von Schadensersatzforderungen und zahlreichen Eingaben konfrontiert, in denen Betroffene die gesundheitlichen Folgen beklagten oder die oftmals schon mit bloßem Auge sichtbaren Verschmutzungen kritisierten. Parteikontrollorgane, wie die Zentrale Kommission für Staatliche Kontrolle (ZKSK), mussten daher im Laufe der 1950er Jahre immer häufiger ausrücken und in lokalen Umweltkonflikten ermitteln. ${ }^{12}$

Die Umweltbelastungen führten aber nicht nur $\mathrm{zu}$ Beeinträchtigungen des Wohlbefindens der Menschen, sondern nahmen immer häufiger auch handfeste volkswirtschaftliche Ausmaße an. Wasserwirtschaftler klagten in den 1950er und 1960er Jahren beispielsweise regelmäßig über Versorgungsprobleme, die durch den Bau neuer Talsperren und großer Verbundnetze alleine nicht behoben werden konnten (Kalweit 1954: 12 f.; Rochlitzer 1960: 634; Musterle 1963: 15). Die Produktion ganzer Industriezweige war aufgrund der großen Abwasserlast gefährdet, die von den ostdeutschen Flüssen schon lange nicht mehr verkraftet werden konnte. ${ }^{13}$ Techniker und Naturwissenschaftler im Umfeld des Amtes für Wasserwirtschaft (AfW), einer zentralen Staatsbehörde, die für die Wasserversorgung von Bevölkerung und Industrie zuständig war, warnten zu Beginn der 1950er Jahre in drastischen Worten davor, dass es zu einem „biologischen Zusammenbruch und nicht zum Aufbau kommen werde", sollten für die vorhandenen Probleme nicht schnellstmöglich Lösungen gefunden werden (Ortleb 1954: 32). In den Gesundheitswissenschaften setzte fast zeitgleich eine Debatte ein, die sich mit den Auswirkungen der Umweltbelastungen auf die Volksgesundheit befasste. Hygieneärzte beklagten die Zunahme von Nebeltagen, die auf Staub- und Schwefeldioxidemissionen der Industrie und den Hausbrand zurückzuführen war und hohe Krankenstände zur Folge hatte. Bronchitiserkrankungen, die nachweislich in Zusammenhang mit den hohen Schwefeldioxidbelastungen standen, nahmen in den 1950er Jahren stark zu. Die Staatliche Hygieneinspektion, eine Exekutivbehörde des Gesundheitsministeriums, sowie die bei den Bezirksräten angesiedelten Hygieneinstitute wurden daher $\mathrm{zu}$ wichtigen Motoren einer gesundheitspolitisch motivierten Umweltreformdebatte (Möller 2018: 82-106).

Die Initiativen von Wasserwirtschaft und Hygiene waren wichtige Bausteine auf dem Weg zu einer sozialistischen Umweltschutzgesetzgebung, blieben zunächst jedoch sehr stark auf sich selbst bezogen. Von entscheidender Bedeutung für die Synthese der unterschiedlichen Reforminitia- 
tiven und somit auch für den Erfolg des umweltpolitischen Aufbruchs war das Engagement einer anderen wissenschaftlichen Akteursgruppe, die von Beginn an sehr viel stärker nach interdisziplinärer Zusammenarbeit und Vernetzung strebte. Der Naturschutz war seit seiner Entstehung im 19. Jahrhundert fest in der Biologie und den Agrarwissenschaften verwurzelt und fand nach der Gründung der DDR in der DAL eine geeignete und einflussreiche institutionelle Plattform, von der aus seine Protagonisten agieren konnten. Die relativ starke Stellung naturpolitischer Anliegen im Forschungsprofil der Akademie spiegelte sich von Beginn an auch in ihren Strukturen wider. Bereits auf seiner ersten Sitzung im Oktober 1951 stimmte das Akademieplenum der Bildung einer Sektion „Landeskultur und Naturschutz" zu, die Behörden und andere staatliche Stellen in Naturschutzfragen beraten, Forschungsarbeiten koordinieren und Netzwerke mit Akteuren anderer für den Naturschutz relevanter Disziplinen knüpfen sollte. Inhaltlich befasste sich die Sektion vorwiegend mit der Weiterentwicklung von Methoden einer „komplexen Standortforschung“, die unter anderem pflanzensoziologische und hydrogeographische Erhebungen umfassten. Des Weiteren erarbeiteten die Naturschutzexperten analog Vorschläge für eine neuartige Form der "komplexen Landeskulturplanung“ und beschäftigten sich mit den Folgen der ländlichen Flurumgestaltungen im Zuge der Kollektivierung der Landwirtschaft. Auch die „Schaffung und Erschließung von Erholungslandschaften" sowie die Beteiligung an der Diskussion um die Einrichtung von Nationalparks in der DDR zählten zum Aufgaben- und Tätigkeitsspektrum. ${ }^{14}$ Im Laufe der 1950er Jahre berichteten auf den Sektionssitzungen außerdem regelmäßig Vertreter benachbarter Disziplinen - darunter Wasserwirtschaftler, Forstwissenschaftler und Sozialhygieniker - von ihrer fachspezifischen Sicht auf Umweltprobleme. Die Arbeit der Sektion trug somit auch entscheidend zur Entstehung einer interdisziplinär vernetzten Problemsicht bei.

Die Sektion war gleich nach ihrer Entstehung an der Ausarbeitung eines Gesetzestextes beteiligt, der das 1935 erlassene Reichsnaturschutzgesetz ersetzen sollte und von der Volkskammer der DDR im August 1954 verabschiedet wurde. Das „Gesetz zur Erhaltung und Pflege der heimatlichen Natur (Naturschutzgesetz)“ regelte administrative Zuständigkeiten innerhalb des Naturschutzes, der nun gänzlich vom SED-Staat inkorporiert wurde, und stellte bis zur Verabschiedung des Landeskulturgesetzes im Jahr 1970 den größten politischen Erfolg der Naturschützer dar. Der wissenschaftliche Redakteur der Zeitschrift „Natur und Heimat“, dem Sprachrohr des inkorporierten Naturschutzes, Reimar Gilsenbach, berichtete noch während der Ausarbeitungsphase im November 1953 über den Gesetzgebungsprozess: 
Die Kommission, die damit beauftragt war, bestand aus Wissenschaftlern verschiedener Fachgebiete, Naturschutzbeauftragten, Justitiaren und Vertretern der Verwaltung. Sie arbeitete eng mit dem Kulturbund zur demokratischen Erneuerung Deutschlands zusammen [...]. So stehen bei der Arbeit am neuen Naturschutzgesetz von Anfang an zwei Momente im Vordergrund: Wissenschaftlichkeit und fortschrittlichdemokratische Auffassung, die den Naturschutz als unerläßliche Notwendigkeit für das gegenwärtige und künftige Wohl des Volkes sieht (Gilsenbach 1953: 350).

Der euphorische Kommentar des Redakteurs gibt einen Einblick in jenen Anpassungsprozess, den Fulbrook als „Normalisierung“ bezeichnet. Der Preis für den Erfolg, den das Naturschutzgesetz zweifelsohne für die Naturschützer darstellte, und die gesteigerte Aufmerksamkeit, die naturpolitischen Interessen infolgedessen zuteilwurde, war die vollständige Inkorporation dieser gesellschaftlichen Klientelgruppe in die sozialistische Herrschaftsordnung. Der Normalisierungsprozess war allerdings keine Einbahnstraße, sondern eröffnete Naturschützern in der DAL und im Kulturbund die Möglichkeit, von innen heraus Einfluss auf Strukturen, Akteure und Entscheidungsprozesse auszuüben. Der Verweis auf die dem Gesetzgebungsprozess zugrundeliegenden Kategorien der „Wissenschaftlichkeit" und der "fortschrittlich-demokratischen Auffassung“, hinter der sich letztlich nichts weiter als ein Bekenntnis zum von der SED energisch vorangetriebenen sozialistischen Aufbau verbarg, veranschaulicht, dass zumindest der überzeugte Sozialist Gilsenbach die wissenschaftliche Naturschutzarbeit auch als ein gleichrangiges Korrektiv dieses Aufbauprojektes verstand.

Das Naturschutzgesetz legte die politische Verantwortung formell in das Ressort des Landwirtschaftsministeriums, das als oberste Naturschutzbehörde fortan dafür verantwortlich war, naturpolitische Interessen im Ministerrat zu vertreten und die Arbeit der mittleren und unteren Naturschutzverwaltungen anzuleiten. Die wissenschaftliche Beraterfunktion, die sich die DAL durch ihre Strukturen de facto bereits selbst zugewiesen hatte, wurde durch das Gesetz ebenfalls festgeschrieben. Die Akademie sollte dafür Sorge tragen, dass die Naturschutzarbeit in der DDR auf eine „wissenschaftliche Grundlage“ gehoben wurde, wie es im Gesetzestext hieß. ${ }^{15} \mathrm{Zu}$ diesem Zweck hatte die DAL bereits ein Jahr vor der Gesetzesverabschiedung ein Institut für Landesforschung und Naturschutz (ILN) in Halle eingerichtet, das bald darauf auch über Zweigstellen in Greifswald, Potsdam, Dessau, Dresden und Jena verfügte und Forschungs- sowie Beratungskompetenzen auf dem Gebiet des Naturschutzes bündelte. Das Forschungsinstitut entwickelte sich zu einer einflussreichen Denkwerkstatt 
und war in den 1960er Jahren maßgeblich an der Formierung der sozialistischen Umweltpolitik beteiligt.

Die Verabschiedung des Naturschutzgesetzes und die verhältnismäßig starke Stellung des Naturschutzes innerhalb des Institutionengefüges der DDR stellten zweifelsohne ein Entgegenkommen der Partei- und Staatsführung gegenüber einer relativ einflussreichen Akteursgruppe dar, die nicht nur über eine einflussreiche Lobby in den Agrarwissenschaften verfügte. Die zahlreichen Naturschutzvereine auf dem Gebiet der DDR, die 1950 in der „Zentralen Kommission für Natur- und Heimatfreunde" unter dem Dach des „Kulturbundes zur demokratischen Erneuerung Deutschlands“ zwangsvereinigt wurden, verschafften dem Naturschutz überdies einen starken gesellschaftspolitischen Rückhalt. Der Kulturbund vertrat Ende der 1950er Jahre immerhin die Interessen von mehr als 45.000 ehrenamtlichen Natur- und Heimatschützern (Behrens et al. 1993: 44). Die enge personelle Verflechtung von naturpolitisch aktiven Wissenschaftlern in der DAL und den „Natur- und Heimatfreunden“ im Kulturbund verlieh daher auch den Initiativen innerhalb der DAL zusätzliches Gewicht.

Die besondere Stellung des Naturschutzes in der Forschungsakademie ging ganz wesentlich auf den Einfluss ihres ersten Präsidenten, Hans Stubbe, zurück. Der Genetiker und Pflanzenzuchtexperte, der nach dem Ende des Zweiten Weltkrieges das Institut für Kulturpflanzenforschung in Gatersleben aufbaute, verfolgte in fachlichen Fragen eine unnachgiebige Haltung und vermochte es, sich den beiden deutschen Diktaturen schnell anzupassen. ${ }^{16}$ Stubbe verfasste bereits 1948 eine Denkschrift, in der er sich für die Reorganisation des Naturschutzes auf dem Gebiet der SBZ einsetzte. ${ }^{17}$ In den folgenden Jahren führte er dieses Engagement im Zusammenwirken mit anderen Wissenschaftlern innerhalb der Akademie fort. Obwohl er sich mit publizistischen und öffentlichen Stellungnahmen zum Naturschutz insgesamt eher zurückhielt, wurde der Netzwerker Stubbe in den 1950er und 1960er Jahren zu einem wichtigen Transmissionsriemen des Naturschutzes. Der sozialistische Wissenschaftsmanager nutzte die restriktiven Mitsprache- und Teilhabemöglichkeiten, die die SED all jenen Akteuren anbot, die dazu bereit waren, sich ihrem Herrschaftsanspruch unterzuordnen, um agrarwissenschaftliche und naturpolitische Interessen durchzusetzen. Bis zum Bau der Mauer profitierte er dabei in besonderem Maße auch von den günstigen Rahmenbedingungen, die die Fluchtmöglichkeit gen Westen dem Wissenschaftspersonal bot. In der Auseinandersetzung um die Übernahme der sogenannten „modernen Agrarbiologie“, die auf die Lehren des Präsidenten der W. I. Lenin-Akademie der Landwirtschaftswissenschaften, Trofim Denissowitsch Lyssenko, zurückging, riskierte er dabei sogar den Bruch mit der SED. Stubbe konnte die Thesen von der Vererbung erworbener Eigenschaften, die Lyssenko in der 
UdSSR durchgesetzt hatte, in seinem Gaterslebener Institut bereits Ende der 1940er Jahre widerlegen. Ein Streit mit der Parteiführung entzündete sich, als der Genetiker den Einfluss dieser Irrlehre im Vorfeld der Gründung der DAL zurückzudrängen versuchte. Das Politbüro beabsichtigte nicht nur Lyssenko zum Ehrenmitglied der Akademie zu machen - ein Umstand, der sich aus politischen Gründen auch kaum verhindern ließ. Die Parteiführung bestand außerdem darauf, dass ein führender Vertreter der "modernen Agrarbiologie“ in der DDR, der Jenaer Biologe Georg Schneider, in das Plenum der DAL aufgenommen werden sollte. Die Personalie Schneider führte zu einer Auseinandersetzung, in der Stubbe zeitweilig den Weggang aus der DDR erwog. Nicht zuletzt aufgrund seiner guten persönlichen Beziehungen konnte er sich in dieser Frage jedoch durchsetzen und Schneider als Plenarmitglied verhindern (Käding 1999: 105-116; Hossfeld \& Olsson 2002: 55 f.; Kuntsche 2014: 341-344).

Die in den 1950er Jahren zunehmenden Versuche einer politischen Vereinnahmung der Wissenschaftslandschaft führten bei Stubbe jedoch nicht zu einer inneren Abkehr von der DDR. ${ }^{18}$ Er scheint in der engen Verflechtung von Politik und Wissenschaft, die durch den Herrschaftsanspruch der SED begründet wurde, vielmehr ein großes Potential für politische Mitgestaltungsmöglichkeiten gesehen zu haben. Stubbe ging es über einzelne Fachfragen hinaus darum, den Einfluss der Wissenschaft auf die Politik zu erhöhen. Dieses Ziel verfolgte er als Präsident der DAL ebenso wie als Vorstandsmitglied des Forschungsrates und als Abgeordneter der Kulturbundfraktion in der Volkskammer, der er von 1963 bis 1986 angehörte. Mit seinem politischen Engagement stand er innerhalb der Gruppe jener Agrarwissenschaftler und Biologen, die sich aktiv für den Naturschutz einsetzten, nicht alleine dar. Auch die Direktoren und Mitarbeiter des ILN, insbesondere Hermann Meusel, Ludwig Bauer und Hugo Weinitschke traten immer wieder mit naturpolitischen Forderungen an sozialistische Funktionsträger und die Öffentlichkeit heran. ${ }^{19}$

Das Verhältnis von Politfunktionären und den Protagonisten des wissenschaftlichen Naturschutzes war jedoch keineswegs rundum harmonisch. Die oberste Naturschutzbehörde, das Landwirtschaftsministerium, betrachtete die naturpolitischen Einmischungen aus der DAL zunehmend als Last. Versorgungsengpässe bei Nahrungsmitteln und die von der SED forcierte Kollektivierung der Landwirtschaft setzten das von der Demokratischen Bauernpartei Deutschlands (DBD) geführte Ministerium immer stärker unter Druck und riefen Unverständnis darüber hervor, dass der Naturschutz innerhalb der Akademiestrukturen eine so starke Stellung genoss. Im September 1960 beschloss das Plenum auf Druck von außen daher die Umbenennung der bisher für Naturschutzfragen zuständigen Sektion in „Landeskultur und Grünland“. Die Stärkung der Grünlandfor- 
schung sollte das von der SED vorgegebene Ziel, die Fleischproduktion zu steigern, umsetzen helfen und laufende Futterbauprogramme unterstützen. Der Naturschutz musste sich damit abfinden, dass seine Interessen innerhalb der Akademie zukünftig nur noch von einer Ständigen Kommission für Landschaftspflege und Naturschutz behandelt wurden. Allerdings bestanden weiterhin enge personelle Verflechtungen zwischen der Sektionsleitung und der neu gebildeten Kommission, so dass die formelle Degradierung des Naturschutzes nicht gleichbedeutend mit einem Gang in die Bedeutungslosigkeit war. ${ }^{20}$

Das belegen auch die Reaktionen führender Naturschutzfunktionäre, die sich unter dem Eindruck des zunehmenden Gegenwinds von Seiten der staatlichen Organe nicht etwa willfährig und kleinlaut, sondern kämpferisch gaben. Im Frühjahr 1959 entzündete sich beispielsweise an einem Flugblatt unter dem Titel „Naturschutz dient dem Arbeiter-und-BauernStaat", das vom Landwirtschaftsministerium herausgegeben worden war, ein offener Schlagabtausch zwischen den Kontrahenten. Nach dem Tod des Chefredakteurs der Zeitschrift „Natur und Heimat“, Karl Kneschke, nutzte Gilsenbach die gesteigerte Aufmerksamkeit, die dem Aprilheft zuteil wurde, um einen publizistischen Gegenangriff zu starten. Im Kern ging es in der darauffolgenden Auseinandersetzung um die Frage, welche Aufgaben dem Naturschutz in der DDR künftig zufallen sollten. Das Ministerium hatte bereits im August des Vorjahres den Versuch unternommen, eine Grundsatzerklärung zu dieser Frage auszuarbeiten, die allerdings von den Naturschützern scharf zurückgewiesen worden war. Das Flugblatt brachte das Fass aus ihrer Sicht schließlich zum Überlaufen. Gilsenbach bezeichnete es als „Hohn auf jede wissenschaftliche Erkenntnis vom Sinn des Naturschutzes" und wies die vom Ministerium vorgesehenen Aufgaben als „Lächerlichkeiten“ zurück (Gilsenbach 1959: 164). Die Polemik Gilsenbachs veranschaulicht, dass die Konformität, in die sich der Naturschutz in der DDR begab, keinesfalls einer bedingungslosen Selbstaufgabe gleichkam. Der Artikel provozierte eine Reihe von Leserbriefen, in denen „Natur- und Heimatfreunde“ der Basis ihre Begeisterung über „derartige ,gepfefferte‘ Darstellungen“ kundtaten. Eine Reaktion des Landwirtschaftsministeriums ließ allerdings nicht lange auf sich warten. In der Juli-Ausgabe der Zeitschrift meldete sich der für das Naturschutzressort zuständige Abteilungsleiter, Fritz Weißhaupt, zu Wort und warf Gilsenbach vor, das von ihm verantwortete Flugblatt nur in Teilen gelesen zu haben. Tatsächlich wies die für solche Schriftstücke unerlässliche ideologische Präambel dem Naturschutz durchaus weitere Aufgaben, etwa im Bereich der Landschaftsgestaltung, zu. Weißhaupt musste in seiner Erwiderung allerdings Fehler einräumen und rechtfertigte die Entscheidung des Ministeriums, die bisher vorliegende Grundkonzeption nicht veröffentlicht 
zu haben, damit, dass zwischen der obersten Naturschutzbehörde, der DAL und der Zentralen Kommission der „Natur- und Heimatfreunde“ im Kulturbund bislang keine Einigkeit über deren Inhalt erzielt werden konnte. Der nun nach außen dringende Dissens zwischen Akteuren, die formal eigentlich an einem Strang ziehen sollten, widersprach dem autoritär-korporatistischen Gesellschaftsverständnis, das der sozialistischen Staatsdoktrin zugrunde lag. Weißhaupt zeigte sich angesichts des leidenschaftlichen Angriffs Gilsenbachs daher darum bemüht, die Gemüter auf Seiten des Naturschutzes zu beruhigen und wieder Sachlichkeit in die Debatte einkehren zu lassen. Der Abteilungsleiter beendete seine Stellungnahme mit einer für die politische Kultur der DDR typischen Aufforderung, in der er an die Naturschützer appellierte, auch weiterhin das Partizipationsangebot des SED-Staates wahrzunehmen (Weißhaupt 1959: 323-325).

Die Vorstellung von Teilhabe, die offiziellen Aussagen wie diesen zugrunde lag, unterschied sich natürlich grundlegend von jenen Partizipationsmöglichkeiten, die Bürgern westlicher Demokratien offenstanden. Dennoch handelte es sich nicht um bloße Lippenbekenntnisse einer totalitären Diktatur. Die SED begriff individuelles Engagement in inkorporierten Organisationen und gesellschaftlichen Kollektiven, die Beteiligung an öffentlichen Diskussionen im Vorfeld von Wahlen, Verfassungsänderungen oder der Verabschiedung von Gesetzen und die politische Kommunikation in Eingaben als wichtige Herrschaftsressourcen, aus denen sie politische Legitimation bezog. Zwar handelte es sich bei diesen Formen von Partizipation um autoritär gelenkte Verfahren, die keinen Raum für Dissens über die politische Grundordnung zuließen (Wolle 1999: 118-121). Abseits der fundamentalen Herrschaftsfragen eröffneten sich in einzelnen Politik- und Gesellschaftsbereichen aber durchaus echte Mitgestaltungsmöglichkeiten für all jene Menschen, die dazu bereit waren, den Zwang zu ideologischer Konformität und die informellen Grenzen der freien Meinungsäußerung zu akzeptieren (Fulbrook 2008: 273-280). Dies galt umso mehr in einer Zeit, als der noch vorhandene Fluchtweg gen Westen immer die Möglichkeit einer Abstimmung mit den Füßen bereithielt.

Für den Naturschutz wirkte der publizistische Schlagabtausch wie ein Befreiungsschlag. Nach dem sich das Landwirtschaftsministerium wieder gesprächsbereit zeigte, erarbeitete die Sektion „Landeskultur und Naturschutz" eine Denkschrift über die "Grundsätze des Naturschutzes in der Deutschen Demokratischen Republik und ihre Verwirklichung", die nun auch von der obersten Naturschutzbehörde akzeptiert wurde. Darin passte sie die Lösung naturpolitischer Probleme sprachlich zwar sehr viel stärker in die sozialistische Ideologie ein. Dafür gelang es den Wissenschaftlern jedoch umgekehrt, ihre Vorstellungen über das Aufgabenspektrum des $\mathrm{Na}$ turschutzes weitestgehend darin zu verankern. ${ }^{21}$ Die Grundsatzerklärung 
bezog aber nicht nur gegen die restriktive Rollenzuschreibung Stellung, die Gilsenbach und andere Naturschützer in den Verlautbarungen des Ministeriums auszumachen glaubten, sondern spiegelte auch einen inneren Wandlungsprozess wider, der sukzessive bereits vor dem Zweiten Weltkrieg innerhalb des Naturschutzes eingesetzt hatte. Schon in den 1930er Jahren vollzogen Naturschützer vielerorts eine Abkehr von älteren, vornehmlich auf die Konservierung von Naturschönheiten bedachten Ansätzen. Die bis dahin dominierende museal-konservierende Naturschutzkonzeption nach Hugo Conwentz wurde von einem neuen, gestalterischen Ansatz verdrängt, der Natur als einen wandelbaren, von Menschen geschaffenen Kulturraum verstand und eine Einbettung von urbanen, industriellen und agrarischen Nutzungsansprüchen mit Hilfe technischer und planerischer Gestaltungsmittel forderte. ${ }^{22}$

Naturschützer verlangten nun ganz selbstverständlich, dass die natürliche Landschaft mehrere Funktionen zugleich erfüllen müsste. Der Schutz von Naturschönheiten und -denkmälern sollte nicht mehr länger nur einem Selbstzweck oder der Bewahrung höherer nationalkultureller Ideale dienen, sondern zum Nutzen des Menschen erfolgen und Reserven sichern, die es zum Wohle des Volkes wirtschaftlich zu nutzen galt. ${ }^{23}$ Die neuen gestalterischen Positionen und Konzepte setzten sich jedoch nicht sofort flächendeckend durch. In den Reihen der ehrenamtlichen Naturschützer dominierte lange Zeit weiterhin ein museal-konservierendes Naturschutzverständnis. Die Arbeit der „Natur- und Heimatfreunde“ konzentrierte sich an der Basis auf das Feld der klassischen Naturschutzarbeit und umschloss vornehmlich die Erfassung, Inventarisierung und den Schutz seltener Arten sowie naturkundliche Vermittlungs- und Erziehungstätigkeiten (Behrens 2001: 42 f.).

Innerhalb des wissenschaftlichen Naturschutzes galten die neuen gestalterischen Anforderungen jedoch bereits zu Beginn der 1950er Jahre als Konsens, auch wenn es weiterhin Kontroversen über deren Umsetzung gab. Unstimmigkeit bestand beispielsweise über die Frage, ob und inwieweit künftig konservierende Elemente in der Landeskultur berücksichtigt werden sollten. Der Gartenbau- und Landschaftsarchitekt Georg Pniower entwickelte in diesem Zusammenhang das Konzept einer „dynamischen Landeskultur“, das sich dezidiert von „rückwärtsgewandten Landschaftsidealen" abgrenzte, die in den 1930er und 1940er Jahren unter dem Einfluss des Nationalsozialismus von führenden Landschaftsarchitekten angewandt wurden. Er trat demgegenüber rigoros für eine Befreiung von überkommenen naturtümelnden und ästhetisierenden Ansprüchen an die Landschaft ein, die seiner Ansicht nach ohnehin erst aus dem Zusammenwirken von Natur und Technik hervorgegangen sei (Behrens 2001: 30-35; Wolschke-Bulmahn \& Fibich 2004: 135-142). Demgegenüber setzte sich Meusel 
erfolgreich für eine Integration von Elementen des konservierenden $\mathrm{Na}$ turschutzes in eine neu zu schaffende Landeskulturplanung ein, ohne dabei die gestalterischen, auf volkswirtschaftliche Nutzeffekte ausgerichteten Erfordernisse infrage zu stellen. Denn auch er betrachtete die natürliche Landschaft Mitteleuropas als ein von Menschenhand geschaffenes Gebilde, unterschied jedoch zwischen einer „alten“, seiner Ansicht harmonischen, und einer „neuen“, durch die Industrialisierung geschaffenen und infolge dessen gestörten Kulturlandschaft (Meusel 1953: 323). Der ILN-Direktor begründete diese Differenzierung aber nicht mit einer an nationalistischen Kulturidealen ausgerichteten Ästhetik, sondern verwies auf naturgesetzliche Zusammenhänge, die in der „alten“ Kulturlandschaft noch ein weitestgehend intaktes Gleichgewicht mit „den Erfordernissen des menschlichen Lebens“ ausbildeten, in der "neuen“ Kulturlandschaft hingegen nur ungenügend berücksichtigt würden. Er zog daher den Schluss, dass eine „fortschrittliche Landeskultur“ sowohl eine „umfassende Untersuchung des Landschaftshaushaltes" voraussetze als auch weiterhin die Einrichtung von Naturschutzgebieten erforderlich mache, die einer „gründliche(n) Landesforschung" als Laboratorien dienten und als Naturreservoire unerlässlich seien (Meusel 1953: 323-326).

Der entscheidende Konnex der Landeskultur blieb in den 1950er Jahren zunächst der auf eine Steigerung der agrarischen Leistungsfähigkeit ausgerichtete Schutz des Bodens. Erst allmählich setzten sich ökologische Konzepte durch, die darüber hinaus auch Wirkzusammenhänge mit anderen Umweltmedien berücksichtigten. Der Gartenbauarchitekt Reinhold Lingner, der zu Beginn der 1950er Jahre ein wissenschaftliches Großprojekt leitete, durch das Umweltschäden auf dem Gebiet der DDR erstmals systematisch kartographiert wurden, plädierte beispielsweise dafür, „die Landschaft als einen Komplex verschiedenartiger Gebilde und Prozesse“ zu verstehen, „die so eng in Wechselwirkung stehen, daß man von einem organischen Zusammenhang sprechen kann." Die einzelnen Landschaftsbestandteile - Wasser, Boden, Luft und die Pflanzendecke - standen seiner Vorstellung nach in einem ständigen Stoffwechsel, „von dessen Ausgeglichenheit der ganze Zustand der Landschaft abhängt. “24 Erst 1958 führte ILN-Direktor Meusel dann eine begriffliche Unterscheidung zwischen der „Landeskultur im engeren Sinne“, die Aufgaben des klassischen Meliorationswesens beinhaltete, und einer "umfassenden Landeskultur" in die Debatte ein, die darüber hinaus auch den Schutz der ökologischen Landschaftsbestandteile beinhaltete (Meusel 1958: 87).

Eine radikale Technikkritik, die noch für ältere Strömungen des Naturschutzes prägend war, fand sich trotz der unterschiedlichen Positionen allerdings weder bei Pniower noch bei Meusel. Letzterer bettete - ganz im Gegenteil - die Landeskultur explizit in das „Zeitalter der Technik“ 
ein, kritisierte allerdings, dass der Einsatz moderner Technik die ökologischen Zusammenhänge des Naturhaushaltes nicht ausreichend berücksichtige und oftmals nur kurzsichtige Interessen verfolge. Meusel plädierte daher für eine umsichtige Planung, die negative Folgen des Technikeinsatzes verhindern und ökologische Aspekte hinreichend berücksichtigen sollte. ${ }^{25}$ Der wissenschaftliche Naturschutz im Umfeld der DAL verlangte nach einer Landeskulturplanung, die nur auf wissenschaftlichen Grundlagen beruhen könne und die Interessen von Industrie, Landwirtschaft, Naturschutz, Wasserwirtschaft und Hygiene so koordinieren sollte, dass künftig das Ausmaß der Umweltschäden möglichst gering gehalten würde. Der Wissenschaft sollte in diesem Zusammenhang die Aufgabe zukommen, geeignete Methoden für den Planungsprozess zu entwickeln und empirische Grundlagen zusammenzutragen. ${ }^{26}$ In diesem Punkt näherten sich die Vorstellungswelten der Naturschützer denen der sozialistischen Machthaber immer stärker an, wodurch ein Konsens zwischen diesen Akteuren erleichtert wurde. Der Naturschutz berief sich außerdem geschickt auf die ideologische Losung „Plane mit, arbeite mit, regiere mit“, die von der SED offensiv propagiert und 1968 auch in Paragraph 21 der neuen Verfassung festgeschrieben wurde, um darauf aufbauend eine Verpflichtung des „Arbeiter-und-Bauern-Staates" zum Schutz der Natur zu postulieren. ${ }^{27}$

\section{Synergieeffekte zwischen Materialökonomie und Naturschutz: Expertennetzwerke und Interessenskoalitionen im Forschungsrat}

In den 1960er Jahren erhielt der Naturschutz zudem Unterstützung aus der UdSSR: Im Oktober 1960 erließ der Oberste Sowjet erstmals ein zentrales Naturschutzgesetz, das von ostdeutschen Naturschützern mit großer Aufmerksamkeit und Genugtuung rezipiert wurde (Weinitschke 1980: 72-74; Würth 1985: 96) ${ }^{28}$ Bereits in den Jahren zuvor beriefen sich Autoren immer wieder auf den sowjetischen Naturschutz, um eigene Forderungen politisch zu legitimieren (Eckhardt 1953: 137-143; Nesmejanow 1958: 136-138). Auch agrarwissenschaftliche Konzepte, deren Reputation auf zweifelhafte Weise zustande kam, wie beispielsweise das Trawopolnaja-Futterbausystem des sowjetischen Bodenkundlers Wassili Robertowitsch Wiljams, wurden von ostdeutschen Naturschützern rezipiert, da sie als politisch opportun galten und der Verwirklichung eigener Interessen dienten. ${ }^{29}$ Der XXII. Parteitag der KPdSU im Jahr 1961, der sich auch mit naturpolitischen Fragen befasste, sowie der zwei Jahre darauf veranstaltete VI. Parteitag der SED, von dem eine große gesellschaftliche Aufbruchsstimmung ausging, verhalfen den naturpolitischen Forderungen zu weiterem Auftrieb. ${ }^{30}$ In den 
folgenden Jahren gelangten reformorientierte Kräfte innerhalb der SED an die Macht und bemühten sich darum, die Zentralverwaltungswirtschaft zu modifizieren. Unter der Bezeichnung „Neues ökonomisches System der Planung und Leitung“ (NÖS) - ab 1967 in einer dritten Etappe als „Ökonomisches System des Sozialismus“ (ÖSS) bezeichnet - versuchten Politfunktionäre und wissenschaftliche Experten bekannte Konstruktionsfehler der Planwirtschaft zu beheben. Anstelle eines rigiden Zentralismus und einer plumpen Fixierung auf die Bruttoproduktion betonten sie das Prinzip der Eigenerwirtschaftung der Finanzmittel und räumten den Unternehmen mehr Eigenverantwortung ein. Während der Reformphase, von der zumindest kurzzeitig auch vorsichtige gesellschaftliche Liberalisierungstendenzen ausgingen, erhielten wissenschaftliche Expertisen und Prognosen über zukünftige Entwicklungsverläufe in verschiedenen Gesellschaftsbereichen eine gesteigerte Aufmerksamkeit. ${ }^{31}$ Die Ständige Kommission für Landschaftspflege und Naturschutz erarbeitete vor diesem Hintergrund „Richtlinien für eine sozialistische Landeskultur", die sich konzeptionell und sprachlich ganz in die Ideenwelt des NÖS fügten und im Dezember 1963, wenige Monate nach dem Parteitag, vom Plenum der DAL bestätigt wurden. ${ }^{32}$

Oberstes Prinzip muß [...] die Erhaltung und Mehrung der in der Natur vorhandenen Reichtümer sein, um die Kulturlandschaft heute und für die Zukunft produktionskräftig, gesund und schön zu erhalten und zu gestalten. Diese als Landeskultur bezeichnete umfassende Aufgabe ist ein System staatlicher und gesellschaftlicher Maßnahmen zum Schutz, zur rationellen Nutzung und zur erweiterten Reproduktion der natürlichen Ressourcen der Kulturlandschaft zum Wohle des Menschen. ${ }^{33}$

Das Papier rückte nicht den Schutz der Natur, sondern den Erhalt und die Nutzung der Kulturlandschaft in den Vordergrund und setzte damit die in den 1930er Jahren eingeschlagene Abkehr von technikkritischen und kulturpessimistischen Positionen fort, die den Naturschutz seit der Jahrhundertwende geprägt hatten (Engels 2006: 36-43). Die ideelle Annäherung an die technischen und ökonomischen Bedürfnisse einer modernen Industriegesellschaft war nicht nur der Einsicht geschuldet, dass ein Zurück in ein vermeintliches Agraridyll oder eine starre Konservierung schützenswerter Naturhabitate flächendeckend weder möglich noch von der Bevölkerungsmehrheit gewollt war. Die Richtlinien stellten ebenso ein deutliches Signal an die SED dar, deren inneres Selbstverständnis untrennbar mit den politischen Leitkategorien eines dauerhaften ökonomischen Wachstums und technologischen Fortschritts verbunden waren. 
Das Papier verstand sich als Entwurf eines naturpolitischen Programmes, das nicht nur darauf abzielte, Ressourcen für die Sphäre des wissenschaftlichen Naturschutzes innerhalb der DAL zu mobilisieren, sondern vielmehr darüber hinaus den Anspruch erhob die Politik der SED so zu beeinflussen, dass die Belange des Naturschutzes gleichberechtigt neben anderen legitimen Zielen stehen und einen festen Platz im Herrschaftsprogramm der SED haben konnten. Gleichzeitig spiegelte das Papier einen inneren Wandlungs- und Anpassungsprozess wider, den der Naturschutz seit den 1950er Jahren ohne eine direkte Steuerung oder harte Repressionen vollzogen hatte.

Die Naturschützer in DAL und ILN warben mit den „Richtlinien für eine sozialistische Landeskultur" um politische Legitimation für ihre Anliegen und begaben sich in den folgenden Jahren auf die Suche nach neuen Fürsprechern, während ihr Verhältnis zur obersten Naturschutzbehörde, deren Funktion nach der Auflösung des Landwirtschaftsministeriums im Jahr 1963 der Landwirtschaftsrat übernommen hatte (Schöne 2010: 46), hingegen anhaltend gestört blieb. Die Wissenschaftler zeigten dabei, dass sie sich nach der Phase der "Normalisierung“ in der neuen Herrschaftsordnung zurechtgefunden hatten und nun in der Lage waren, selbstständig alternative politische Kanäle zu aktivieren. Im Jahr 1963 erhielten Mitarbeiter des ILN - vermutlich Dank der Fürsprache Stubbes - die Möglichkeit, ihr Anliegen vor der Volkskammer zu präsentieren. Institutsdirektor Meusel und sein wissenschaftlicher Mitarbeiter, Hugo Weinitschke, referierten auf einer eigens einberufenen Plenarsitzung über die „Aufgaben der Landeskultur beim Aufbau des Sozialismus" und bemühten sich darum, die Abgeordneten zu einer Resolution zu bewegen, in der der Staatsrat da$\mathrm{zu}$ aufgefordert werden sollte, die noch in der Ausarbeitung befindlichen Richtlinien für eine sozialistische Landeskultur rechtskräftig zu beschließen. ${ }^{34}$ Zwar scheiterte dieses Ansinnen vorerst, doch bereits ein Jahr darauf gelang es, vermutlich abermals dank Stubbes Einfluss, ein Treffen zwischen den Präsidenten der DAL und der AdW sowie Volkskammerpräsidenten Dieckmann anzuberaumen, bei dem die Forderungen des Naturschutzes nochmals erörtert wurden (Weinitschke 1963: 33). ${ }^{35}$ Die Sektion „Landeskultur und Grünland“ erarbeitete im Vorfeld dieser Zusammenkunft eine Neufassung der Richtlinien, die jetzt sehr viel entschiedener nach einem Landeskulturgesetz verlangten, das das Naturschutzgesetz aus dem Jahr 1954 ersetzen sollte, und gleichzeitig eine Brücke zu den Forderungen anderer Umweltreforminitiativen, etwa der Hygiene, schlug. ${ }^{36}$

Die Beharrlichkeit der Naturschützer hatte Erfolg, auch wenn sich dieser nur nach und nach einstellte: Im April 1966 unterstützte Dieckmann auf einer Pressekonferenz anlässlich der zehnten Naturschutzwoche öffentlich die Forderungen des Naturschutzes und bezeichnete alles bis dahin Geta- 
ne als ungenügend. Unter Verweis auf das sowjetische Naturschutzgesetz forderte er „auch in der Deutschen Demokratischen Republik wirksame Maßnahmen zur Durchsetzung der Prinzipien sozialistischer Landeskultur zu ergreifen." (Dieckmann 1966: 67 f.) Herbert Weiz, Staatssekretär für Forschung und Technik, und SED-Kulturfunktionär Alexander Abusch setzten sich nun ebenfalls sowohl beim Vorsitzenden des Ministerrates, Willi Stoph, als auch bei Gerhard Schürer, dem Vorsitzenden der Staatlichen Plankommission (SPK), für die Forderungen des Naturschutzes ein. ${ }^{37}$ Schürer, von dem man sich erhoffte, dass er die SPK zu einer zentralen Koordinationsstelle für die Aufgaben der „sozialistischen Landeskultur“ machen würde, erteilte diesem Anliegen zwar eine Absage, sprach sich im September 1968 anlässlich der Vorbereitung des kommenden Perspektivplanes aber entschieden für mehr Investitionen in den Umweltschutz aus. $^{38}$

Der Schlüssel für die Verankerung natur- und umweltpolitischer Ziele auf der politischen Ebene des SED-Staates lag jedoch im Forschungsrat, dessen Einflussmöglichkeiten in der späten Ulbricht-Ära einen Höhepunkt erreichten. Der wissenschaftspolitische Beirat wurde während der Phase des NÖS/ÖSS zu einer wichtigen Plattform, um die ökonomischen Ziele der Reformen mit den Forderungen des Naturschutzes und anderer Umweltschutzinitiativen zu vereinen. In den 1960er Jahren entstanden dort gleich mehrere Kommissionen, die an der Lösung umweltpolitischer Teilfragen arbeiteten. ${ }^{39}$ Stubbe hatte die Bedeutung des Gremiums für den Naturschutz früh erkannt und sich bereits im Frühjahr 1960, kurz nachdem der Streit mit dem Landwirtschaftsministerium formell beigelegt worden war, hilfesuchend an den Forschungsratsvorsitzenden, Peter Adolf Thiessen, gewandt. In einem Schreiben beklagte er, dass „unsere vielfachen mahnenden und beschwörenden Darlegungen zu den genannten Problemen [...] bisher praktisch ungehört verhallt" seien und es an der Spitze bislang an einem Mann fehle, der „den Ernst der Situation erkennt und mit allem Nachdruck an der Verwirklichung der Aufgaben von Landesforschung und Naturschutz arbeitet." 40

Eine Gelegenheit, diese prekäre Situation zu überwinden, eröffnete sich den Naturschützern fünf Jahre später. Im Februar 1965 konstituierte sich innerhalb des Forschungsrates eine Kommission, die den Auftrag erhielt, eine "Grundkonzeption für die Behandlung industrieller Abprodukte" zu erarbeiten. ${ }^{41}$ Den Vorsitz über das Expertengremium übernahm der Ökonom Helmut Koziolek, der zu den wissenschaftlichen Beratern des SEDReformflügels um Walter Ulbricht, Erich Apel, Günter Mittag und Wolfgang Berger gehörte und kurz darauf zum Direktor des neugegründeten Zentralinstituts für Sozialistische Wirtschaftsführung beim ZK der SED berufen wurde (Kaiser 1997: 62; Malycha \& Winters 2009: 170 f.). Der 
Grund für die Bildung der Kommission war die starke Zunahme von Abprodukten aus der industriellen Produktion, insbesondere von Braunkohlenasche, die täglich tonnenweise bei den neu errichteten Großkraftwerken anfiel und deren Entsorgung nicht nur schwer lösbare technische Probleme, sondern auch hohe Kosten verursachte (Möller 2018: 165-168). Konzeptionell knüpfte die Arbeit der Expertenkommission an etablierte Praktiken einer autarkistischen Altstoffverwertung an, deren Ursprünge bis in die Zeit des Nationalsozialismus zurückreichten sowie an Vorüberlegung ostdeutscher Materialökonomen, die Altstoffe als eine sekundäre Rohstoffbasis betrachteten, die angesichts der prekären Rohstoffsituation der DDR gezielt für die Verwirklichung wirtschaftspolitischer Wachstumsziele nutzbar gemacht werden sollte (Möller 2014: 67-79).

Stubbe erkannte schnell das große Potential, das von der AbprodukteKommission und der ihr zugrundeliegenden Arbeitskonzeption ausging. Nach der konstituierenden Sitzung beeilte er sich an Meusel zu berichten, dass „diese Kommission [...] mir für alle Fragen der Landeskultur und des Naturschutzes von größter Bedeutung zu sein“ scheine, „denn sie hat sich auch mit dem Problem der Schäden zu befassen, die durch industrielle Abprodukte in der Landwirtschaft entstehen, ebenfalls auch mit Rauchschäden und vor allem auch mit der Verwertung industrieller Abprodukte.“ Der DAL-Präsident nutzte das erste Zusammentreffen außerdem, um „sofort die Frage der Standorte neuer Industrieanlagen und deren Beurteilung von verschiedenen Seiten“ anzuschneiden „und gleichfalls wieder auf die katastrophale Verunreinigung der Flüsse, die Rauchschäden, die Notwendigkeit der Erholungsgebiete etc." hinzuweisen. ${ }^{42}$ An der Erarbeitung der Grundkonzeption waren Mitarbeiter des ILN, das im September 1965 von Stubbe als Vertreterinstitut der DAL in der Kommission eingesetzt wurde und intern die Koordinierung der Zuarbeiten weiterer Akademieinstitute übernahm, maßgeblich beteiligt. ${ }^{43}$

Die Kommission, die bereits im Frühjahr 1966 ein Ergebnis vorlegen konnte, setzte die Grundlagen für die sich bald darauf formierende ostdeutsche Umweltpolitik. Dreh- und Angelpunkt der technokratischen Konzeption war der Gedanke, mithilfe von Abfallverwertung und -vermeidung den Schadstoffausstoß bereits im Produktions- und Konsumtionsprozess $\mathrm{zu}$ reduzieren und auf diese Weise gleichzeitig ökonomische Nutzeffekte $\mathrm{zu}$ erzielen und die Folgekosten der Umweltverschmutzungen zu verringern (Möller 2015: 151 f.; Möller 2018: 187-198). Dieser Ansatz, der in den 1970er Jahren im Zuge der Diskussion über die "Grenzen des Wachstums“ auch im Westen an Bedeutung gewinnen sollte (Hünemörder 2004; Köster 2017), wurde in den folgenden Jahren fortentwickelt. Den Initiativen des wissenschaftlichen Naturschutzes und anderer Umweltreformkräfte kam dabei eine gesellschaftliche Entwicklung zugute: Seit Beginn der 1960er 
Jahre nahmen Eingabenproteste aus der Bevölkerung gegen die wachsenden Umweltbeeinträchtigungen stark zu. Eine abermalige Häufung von Protestbriefen in der ersten Jahreshälfte setzte 1967 auf den Ebenen des Staats- und Ministerrates eine Dynamik in Gang, die dem Umweltschutz schlagartig zu großer politischer Aufmerksamkeit verhalf (Möller 2018: 173-187). Im Oktober des Jahres beauftragte der Ministerrat eine Arbeitsgruppe unter der Leitung des DBD-Funktionärs Werner Titel damit, eine Prognose über „Industrielle Abprodukte und die planmäßige Gestaltung einer sozialistischen Landeskultur" zu erarbeiten, die die Vorarbeiten innerhalb der DAL und des Forschungsrates begrifflich zusammenführte. Bereits ein Jahr darauf legte Titel ein weit über 100 Seiten umfassendes Konzept vor, das als umweltpolitische Planungsgrundlage für die beiden kommenden Fünfjahrespläne der 1970er Jahre herangezogen werden sollte. Auch an der Entstehung dieses Dokumentes war das ILN unter der Federführung von Hugo Weinitschke maßgeblich beteiligt. ${ }^{44}$

Die bereits einige Monate zuvor in Kraft getretene neue Verfassung enthielt nun als Konsequenz dieser Entwicklung in Artikel 15 einen Passus, der „die Reinhaltung der Gewässer und der Luft sowie der Schutz der Pflanzenund Tierwelt und der landschaftlichen Schönheiten der Heimat" zur Staatsaufgabe erklärte (Verfassung 1968: 16). Im Mai 1968 verabschiedete der Bezirkstag Halle außerdem einen Beschluss zur Reinhaltung der Luft und der Gewässer, der konkrete Vorschläge für ein erstes Maßnahmenpaket unterbreitete und die Rahmenbedingungen für ein „ökonomisches Experiment" feststeckte, in dem in den darauffolgenden beiden Jahren wichtige Elemente der neuen Umweltpolitik erprobt werden sollten. ${ }^{45}$ Die Verabschiedung des Landeskulturgesetzes im Mai 1970 war eine direkte Folge dieser Dynamik, wenngleich das neue Umweltrahmengesetz im Kern auf das beharrliche Engagement zurückzuführen war, das der ostdeutsche Naturschutz seit den späten 1950er Jahren entfaltete.

\section{Funktionalisierung als erfolgreiche Legitimationsstrategie und Verhängnis des Naturschutzes}

Die kurze Phase des umweltpolitischen Aufbruchs, die 1967 einsetzte und bereits ab Mitte der 1970er Jahre infolge des Machtwechsels von Ulbricht zu Honecker und einer aufziehenden ökonomischen Krise wieder abebbte, stand ganz im Zeichen jenes Konsensprinzips, das für die Herrschaft der SED konstitutiv war (Möller 2018: 258-260; Sabrow 2001: 418-421). Die Partei- und Staatsführung ließ öffentliche Debatten über den Zustand der Umwelt und notwendige Lösungsmaßnahmen zu und förderte diese 
sogar zeitweise, indem bestehende Veranstaltungsformate, wie die Naturschutzwochen, zu landesweiten „Wochen der sozialistischen Landeskultur" ausgebaut und neue Institutionen geschaffen wurden. Die SED griff das neue Umweltschutzthema zunächst bereitwillig auf und versuchte es auf nationaler wie internationaler Bühne für ihre politischen Interessen zu nutzen, ehe diese Verlautbarungen im Zeichen des wirtschaftlichen und gesellschaftlichen Verfalls in den 1980er Jahren zu ideologischen Durchhalteparolen und Lippenbekenntnissen verkamen (Möller 2018: 260-280, 293-299).

Der wissenschaftliche Naturschutz wurde im Zuge dieses Aufbruchs immer stärker in die neue sozialistische Umweltpolitik eingebunden. Schon die Naturschutzwoche 1969 machte dies deutlich: Der Hauptredner der Eröffnungsveranstaltung in Zwickau, Werner Titel, beschwor eine Konsensformel, wonach „das System der sozialistischen Landeskultur“ nur ein „immanentes Teilsystem des entwickelten gesellschaftlichen Sozialismus" sein könnte und in diesem Sinn als „sehr komplexe gesellschaftliche Aufgabe“ verstanden werden müsse, „die in allen Wirtschaftsbereichen zu lösen ist, um die grundlegenden natürlichen Existenzbedingungen der Gesellschaft zu sichern." (Titel 1969: 14) Gleichzeitig betonte er, dass es notwendig sei, „den Erfahrungsaustausch und die Diskussion über die notwendigen und unter den Bedingungen der Volkswirtschaft der DDR möglichen Maßnahmen für eine zweckmäßige und planmäßige Gestaltung der sozialistischen Landeskultur unserer Republik zu führen und eine breite Mobilisierung der Öffentlichkeit, ihre Konzentration auf die Schwerpunkte der Landeskultur zu fördern." (Titel 1969: 22).

Die führenden Vertreter des wissenschaftlichen Naturschutzes verstanden Aussagen wie diese als ein Entgegenkommen der Politik und akklamierten ganz im Sinne des geltenden Konsensprinzips umgekehrt dem Beitrag des stellvertretenden Vorsitzenden des Ministerrates. Meusel zeigte sich „glücklich, hier einmal das ausgesprochen zu hören, was wir in kleinen Kreisen der Natur- und Heimatfreunde, in kleinen Kreisen der Wissenschaftler schon so häufig besprochen und diskutiert haben." (Meusel 1969: 38) Weinitschke machte als Antwort auf die Frage danach, was „wir in den letzten 15 Jahren erreicht“ haben, vor allen Dingen „eine geistige Wandlung, eine grundsätzliche neue Bewußtseinsbildung bei vielen $\mathrm{Na}$ turschützern“ aus: „Heute sind wir uns klar darüber, daß der umfassende Naturschutz, im Interesse aller Menschen, in der rationellen, planmäßigen Nutzung und damit auch in der Pflege der Natur und ihrer Reichtümer besteht." (Weinitschke 1969: 27 f.).

Der von Weinitschke aus Sicht eines inkorporierten Funktionsträgers beschriebene Anpassungsprozess an die neuen Herrschaftsverhältnisse, den Fulbrook als Phase der „Normalisierung“ beschreibt, war eine grundle- 
gende Voraussetzung für den umweltpolitischen Aufbruch und die Verabschiedung des Landeskulturgesetzes. Die Indienststellung naturpolitischer Konzepte in die politische Ökonomie und die Herrschaftsideologie der SED eröffnete Naturschützern den Zugang zu politischen Ressourcen. Der Preis dafür war die Hinwendung zu einer Ökologie, die (material)ökonomische Nutzeffekte stärker gewichtete als biologische Zusammenhänge, und deren Erfolg maßgeblich an ein kontinuierliches Wirtschaftswachstum sowie stete technologische Innovation geknüpft war.

Die Naturschützer innerhalb der DAL beziehungsweise des ILN wurden in den folgenden Jahren immer enger in die entstehenden Strukturen einer neuen sozialistischen Umweltpolitik eingebunden und verloren sich in den 1970er Jahren in der Kärrnerarbeit eines angepassten und funktionalisierten Umweltschutzes, dessen Handlungsmöglichkeiten angesichts der sich zuspitzenden ökonomischen Krise schrumpften (Möller 2018: 260-278). Diese Verwicklung mit der Macht wurde den Vertretern des Natur- und Umweltschutzes zum Verhängnis: Als sich Ende der 1970er Jahre, getragen von erneut aufflammenden Eingabenprotesten und neuen Akteuren, wie etwa den oppositionellen Umweltgruppen unter dem Dach der Evangelischen Kirchen oder den kritischen Jugend- und Stadtökologiegruppen in der staatsnahen Gesellschaft für Natur und Umwelt, eine Neuaushandlung der Umweltfrage anbahnte, erschienen die etablierten, konsensual agierenden Umweltwissenschaften - von einigen Ausnahmen abgesehen merkwürdig stumm.

\section{Anmerkungen}

1 So beispielsweise Wolfgang Harich, Rudolf Bahro und Robert Havemann. Eine Analyse der drei „marxistischen Systemkritiker" bei Amberger (2014).

2 Gesetz über die planmäßige Gestaltung der sozialistischen Landeskultur in der Deutschen Demokratischen Republik - Landeskulturgesetz - vom 14. Mai 1970, in: GBl. DDR, I, 67-74.

3 Präsidium des Ministerrates, Beschluß über weitere Maßnahmen zur Leitung und Planung der sozialistischen Landeskultur und des Umweltschutzes vom 20.09.1972: BArch, DK 5/1971.

4 Diese Einschätzung ging ursprünglich auf eine journalistische Reportage aus den $1980 \mathrm{er}$ Jahren zurück und wurde seitdem immer wieder unhinterfragt reproduziert. Vgl. Wensierski (1986: 49). Zur Reproduktion vgl. exemplarisch: Hünemörder (2004: 262 f.); Huff (2015: 166-177).

5 Parallel dazu weitete die Partei- und Staatsführung die Kontrolle über die neue wissenschaftliche Institution aus: Nachdem die Einrichtung 1949 für kurze Zeit dem Ministerium für Volksbildung untergeordnet war, fasste das Politbüro der SED zu Beginn der 1950er Jahre einen Beschluss, durch den die DAW künftig direkt dem Ministerrat unterstellt sein sollte. Der etwa zeitgleich einsetzende Rückzug der Sowjetischen Kontrollkommission von wissenschaftspolitischen Steuerungsansprüchen ermöglichte es der Partei außerdem, die Akademie nun in den Einflussbereich der 1952 gebilde- 
ten ZK-Abteilung für „Wissenschaft und Hochschulen“ zu rücken und auf diese Weise gezielter zu kontrollieren und anzuleiten. Vgl. Kocka et al.( 2002: 367, 406 f.); Laitko (2014: 17, 29 f.).

6 Die Bedeutung des Forschungsrates scheint allerdings infolge des Machtwechsels von Ulbricht zu Honecker deutlich abgenommen zu haben. In den 1960er Jahren war es dem Gremium allerdings möglich, auch ohne den Umweg über die in vielen Bereichen noch schwach entwickelte Parteibürokratie entscheidenden Einfluss auf höchste politische Instanzen auszü̈ben. Wagner (1992: 76 f., 204 f., 248-251); Tandler (2000: 240-245; Malycha 2005: 189 f.)

7 Die 1972 in Akademie der Wissenschaften der DDR (AdW) umbenannte Institution verfügte nun über große Zentralinstitute und wies kaum noch Eigenschaften der einstmaligen preußischen Gelehrtengesellschaft auf. Auch die inneren Steuerungsmechanismen der Akademie veränderten sich: Über das Vorschlagsrecht für die Zuwahl neuer Wissenschaftler verfügten seit Beginn der 1970er Jahre neben den ordentlichen Mitgliedern auch der Ministerrat und das Präsidium des Forschungsrates, so dass es der Partei- und Staatsführung möglich war, direkten Einfluss auf die personelle Zusammensetzung der DAW zu nehmen. Vgl. Tandler (2000: 129-131); Kocka et al. (2002: 383-388); Malycha (2005: 191-196).

8 Zum Zitat: Meuschel (1992, 10, 186 f.); vgl. auch Förtsch (1997: 25); Connelly (1997: 103 f.); Jessen (2005, 249-254).

9 Vgl. exemplarisch Büschenfeld (1997); Uekötter (2003); Hünemörder (2004); Engels (2006); Hasenöhrl (2011).

10 Auf die Existenz früher Initiativen hat Gerhard Würth bereits Mitte der 1980er Jahre in einer gemessen am Entstehungshintergrund erstaunlich detail- und kenntnisreichen Literaturstudie hingewiesen. Würth (1985: 23-31). Ausführlicher dazu und auf Basis umfangreicher Archivrecherchen: Möller (2018: 54-133).

11 Zentrale Kommission für Staatliche Kontrolle, Arbeits-Anweisung, Berlin, am 05.04.1954: BArch, DC 1/636, Teil 1 von 2. Zum Volksaufstand und zum Neuen Kurs vgl. exemplarisch Weber (2000: 41 f.); Hoffmann (2013: 38-45).

12 Die umfangreichen Untersuchungsberichte der Kontrollkommission dokumentierten anschaulich den wachsenden Problemdruck. Möller (2018: 57-68).

13 So beispielsweise in der Zellstoffindustrie, deren Betriebe als Umweltsünder und Geschädigte in besonderem Maße in die Debatte involviert waren. Möller (2018: $66 \mathrm{f}$.).

14 Vgl. dazu exemplarisch DAL, Sektion Landeskultur und Grünland, Jahresbericht 1960 der Sektion Landeskultur und Grünland, Berlin, den 19.01.1961: BArch, DK 107/5397, pag. 174-178. Zur politisch aufgeladenen und durchaus konfliktbehafteten Diskussion um die Einrichtung von Nationalparks vgl. exemplarisch Wiedemann, (1958a: 152-155) und Wiedemann (1958b: 176-178) sowie allgemein Behrens (2005: 64-69).

15 Gesetz zur Erhaltung und Pflege der heimatlichen Natur (Naturschutzgesetz), in: GBl. DDR, I, 1954, 695-698, hier \$ 13, 697.

16 Nach eigener Darstellung erfuhr Stubbes Karriere Mitte der 30er Jahre einen Dämpfer, weil er sich für einen jüdischen Kollegen am Kaiser-Wilhelm-Institut für Züchtungsforschung in Müncheberg eingesetzt hatte. Dieses Engagement, das ihm nach 1945 als Leumund für eine antifaschistische Gesinnung diente, darf aber nicht darüber hinwegtäuschen, dass er in vielerlei Hinsicht eine unbekümmerte Nähe zum Nationalsozialismus zeigte, die noch in den 60er Jahren durch sein öffentlich vorgetragenes Engagement für eine "freiwillige Eugenik“ sowie einzelne Episoden, wie etwa den Einsatz für einen befreundeten Kollegen, der als Mitglied der Waffen-SS im Konzentrationslager Auschwitz tätig war und von Stubbe im Verfahren vor einem polnischen Gericht einen „Persilschein“ erhalten hatte. Käding (1999: 36-47); Heim (2003: 209-211, 217 f); Gudermann (2006: 175 f.).

17 Entwurf, Denkschrift über die Reorganisation des Naturschutzes in der Sowjetischen Besatzungszone Deutschlands (Januar 1948) zitiert nach Stubbe (2002: 113). Vgl. dazu auch Käding (1999: 150 f.). 
18 Auch in der Affäre um den SED-Agrarfunktionär Kurt Vieweg oder in seinem Einsatz gegen die Übernahme der sowjetischen "Quadratnestmethode“ im Feldfruchtanbau stellte er sich gegen die Parteilinie, ohne jedoch mit der SED zu brechen. Stubbe verstand Wissenschaft als ein Korrektiv der Politik und beklagte vor dem Hintergrund der Auseinandersetzungen eine „Vertrauenskrise zwischen Wissenschaft und Staatsapparat", die seiner Ansicht nach durch die Nichtbeachtung wissenschaftlicher Expertise heraufbeschworen wurde. Der politisch-ideologische Konformismus, den er dennoch dabei an den Tag legte, und seine wissenschaftliche Reputation halfen ihm, politische Konflikte unbeschadet zu überstehen und ermöglichten es, dass er sich in einzelnen Fragen auch immer wieder durchsetzen konnte. Vgl. dazu: Schreiben von Akademiepräsident Hans Stubbe an den SED-Landwirtschaftssekretär Erich Mückeberger wegen der Abberufung von Kurt Vieweg aus Ämtern in der Akademie der Landwirtschaftswissenschaften, 15.03.1957, sowie Rede von Akademiepräsident Hans Stubbe in der Agrarkommission des Politbüros der SED am 26.02.1960 (Auszug), beide in: Kuntsche (2017, $434-435,450-458$, insbes. 453 f.).

19 So etwa im Juli 1964 in einer Beilage des SED-Blattes Neues Deutschland: Bauer et al. (1964), Auch die Luft will gepflegt sein, in: ND-Beilage Nr. 27 vom 4. Juli 1964, 5.

20 Nach der offiziellen Abschaffung der Lebensmittelkarten 1958 gelang es nicht, die landwirtschaftliche Produktion ausreichend zu steigern, so dass es bereits nach kurzer Zeit zu neuen Rationierungen kam. Vgl. Stefan Wolle, Der große Plan. Alltag und Herrschaft in der DDR 1949-1961, Berlin 2013, 374-385. Zur Umbenennung der Sektion und den politischen Hintergründen vgl.: DAL, Sektion Landeskultur und Grünland, Jahresbericht 1960 der Sektion Landeskultur und Grünland, Berlin, den 19.01.1961: BArch, DK 107/5397, pag. 170 f.; Schöne (2010: 194-198).

21 Das wurde insbesondere bei der Auflistung der „Aufgaben zur Erhaltung und Pflege der heimatlichen Natur" deutlich, die stellenweise eher den Charakter programmatischer Forderungen annahmen: Grundsätze des Naturschutzes in der Deutschen Demokratischen Republik und ihre Verwirklichung, Berlin, den 05.01.1960: BArch, DK 107/7658. Vgl. auch Stubbe an den Vorsitzenden des Forschungsrates, Herrn Professor Dr. Thiessen vom 15. Januar 1960: BArch, DK 107/7658.

22 Mit diesem Wandel folgten die ostdeutschen Naturschützer auch einem internationalen Trend. Der britische Biologe und erste Generaldirektor der UNESCO, Julian Huxley, forderte bereits in den 1930er Jahren zusammen mit anderen Ökologen die Einführung staatlicher Steuerungsinstrumente, mit deren Hilfe eine gezielte Bevölkerungsund Raumplanung im Sinne des Naturschutzes ermöglicht werden sollte. Radkau (2011: 104-108); Wöbse (2012: 278-287, insbes. 282 f.). Für die Entwicklung im Nationalsozialismus und in der Bundesrepublik vgl.: Zeller (2003: 273-307, hier 275-281); Oberkrome (2005: 23-37, hier 29-34); Hasenöhrl (2011: 71 f.).

23 Dieser Wandel wurde unter anderem in den ambitionierten, in der Praxis aber nur bedingt umgesetzten Konzepten der sogenannten „Landschaftsanwälte“ um den Landschaftsarchitekten Alwin Seifert deutlich: Zeller (2003: 273-307, hier 275-281). Zum konzeptionellen Wandel in den 1930er Jahren vgl. Oberkrome (2005: 23-37, hier 29-34). Andere Autoren verorten diesen Wandel stärker in den 1950er Jahren: Vgl. Würth Umweltschutz (1985, 91 f.); Rösler et al. (1990: 7); Hasenöhrl Zivilgesellschaft (2011, 71 f.). Für die DDR vgl. exemplarisch: Goetel (1959: 164).

24 Beide Zitate aus: Lingner $(1952,7)$.

25 Diese Denkweise wird insbesondere auch bei Gilsenbach deutlich, der am Ende seiner populärwissenschaftlichen Mahnschrift "Die Erde dürstet" voller Bewunderung und Optimismus auf die großen sowjetischen Wasserbauprojekte in Sibirien - den sogenannten „Dawydow-Plan“ - und die Vision Hermann Sörgels, der in den 1920er Jahren vorschlug, an der Straße von Gibraltar einen gigantischen Staudamm zu errichten, hinwies. Vgl. Gilsenbach (1961: 287-294).

26 Ebd., 94-97. Ähnlich bei: Lingner (1952: 75 f.); Kritischer bei: Gilsenbach (1959: 163 f.). 
27 Grundsätze des Naturschutzes in der Deutschen Demokratischen Republik und ihre Verwirklichung, Berlin, den 05.01.1960: BArch, DK 107/7658.

28 Zur Wahrnehmung vgl. auch: Das neue Naturschutzgesetz in der Sowjetunion, von Dr. H. Hiebsch: BArch, DK 107/7658; DAL, Ständige Kommission für Landschaftspflege und Naturschutz, Grundsätze für eine der sozialistischen Gesellschaftsordnung entsprechende Gebietsplanung und Landeskultur, Berlin, d. 5. Dez. 1963: BArch, DK 107/5398, pag. 83.

29 Die Lehren Wiljams, der unter anderem eine besondere Form der Fruchtfolge im Feldgrasanbau und die Anlage von Gehölzstreifen propagierte, wurden in den 1930er Jahren - ähnlich wie im Fall der Lehren Lyssenkos - mit Hilfe stalinistischer Gewalt zur Staatsdoktrin erklärt und fanden daraufhin auch eine weite Verbreitung in den Ostblockstaaten. Naturschützer plädierten unter anderem deshalb für die Anwendung des Trawopolnaja-Systems, da es die systematische Anlage breiter Hecken- und Gehölzstreifen vorsah. Vgl. Nikonow \& Schulze (2004: 23 f., 79-81). Vgl. zur Rezeption: Pniower (1953: 108); Meusel (1953: 324).

30 Die Hintergründe der Verabschiedung des neuen Naturschutzgesetzes und der Diskussionen auf dem XXII. Parteitag der KPdSU werden im Hinblick auf die Zustimmung der politischen Führungsebene um Chruschtschow zum Naturschutz von der Forschung bislang allerdings zurückhaltend eingeschätzt: Vgl. Gestwa (2010: 508-510).

31 Zur Geschichte der Wirtschaftsreform vgl.: Roesler (1990); Steiner (1999); Meuschel (1992: $181 \mathrm{ff} ., 190,192-211)$.

32 DAL an alle Mitarbeiter der Sektion Landeskultur und Grünland und an alle Mitglieder der Ständigen Kommission für Landschaftspflege und Naturschutz vom 03.05.1962 sowie Gesichtspunkte zur Ausarbeitung von „Richtlinien für eine sozialistische Landeskultur“, 03.05.1962: BArch, DK 107/7658.

33 DAL, Ständ. Kommission für Landschaftspflege und Naturschutz, Grundsätze für eine der sozialistischen Gesellschaftsordnung entsprechende Gebietsplanung und Landeskultur, Berlin, d. 5. Dez. 1963: BArch, DK 107/5398, pag. 83.

34 Nach Artikel 106 des Gesetzes über die Bildung des Staatsrates der DDR war es dem kollektiven Staatsoberhaupt möglich, Beschlüsse mit Gesetzeskraft zu verabschieden. Vgl. Gesetz über die Bildung des Staatsrates der Deutschen Demokratischen Republik vom 12. September 1960, in: Gesetzblatt der DDR, Teil I, 1959, 89.

35 Koriath an Bauer, 16.06.1964: BArch, DK 107/2967. Zum Scheitern der Volkskammerinitiative: Obwohl die anschließende Debatte den Eindruck vermittelte, dass die von Meusel und Weinitschke gestellten Forderungen und sogar die Verabschiedung eines neuen, weitergehenden Naturschutzgesetzes nur noch eine Formfrage gewesen wären, scheiterte die Volkskammer-Initiative ein Misserfolg. Von 469 Abgeordneten erschien weniger als ein Zehntel zur Plenarsitzung und auch das eigentliche Ziel, die Erwirkung einer Resolution, erreichten die Wissenschaftler nicht. Die Naturschützer ließen sich jedoch nicht beirren und drängten weiterhin darauf, politische Fürsprecher für ihre Ziele politisch zu gewinnen. Meusel wertete die Initiative bereits in einem Schreiben, dass er wenige Tage nach der Volkskammersitzung an Stubbe richtete, als Erfolg: Meusel an Stubbe, 4. Juni 1963: BArch, DK 107/2967.

36 Entwurf einer Erklärung über die Prinzipien sozialistischer Landeskultur (Neufassung vom 16.07.1964): BArch, DK 107/2967.

37 Die Initiative ging auf eine Empfehlung der Gruppe Land- und Forstwirtschaft des Forschungsrates vom 07.01.1966 zurück: Der Staatssekretär, Dr. Weiz an Genossen Minister Schürer, 14.03.1966; Schürer an Staatssekretariat für Forschung und Technik, 29.04.1966; Weiz an Stellvertreter des Vorsitzenden des Ministerrates der DDR, Genossen Abusch, Berlin, 06.10.1966; Abusch an Vorsitzenden des Ministerrates, Genossen Stoph, Probleme der sozialistischen Landeskultur, 24. Oktober 1966; alle aus: BArch, DC 20/19119, pag. 3-13. 
38 Gerhard Schürer, Bericht des Vorsitzenden der Staatlichen Plankommission über den Stand der Vorbereitung des Perspektivplanes (gekürzte Fassung), 25. September 1968: SAPMO, DY 3023/450, pag. 51.

39 So beispielsweise die einflussreiche Kommission „Reinhaltung der Luft“ unter der Leitung des Hygienewissenschaftlers und Begründers der ostdeutschen Umweltmedizin Karlwilhelm Horn sowie eine Kommission zur Behandlung umweltrelevanter Probleme in der Wasserwirtschaft. Vgl. Möller 2018: $148 \mathrm{f}$.

40 Stubbe an den Vorsitzenden des Forschungsrates, Herrn Professor Dr. Thiessen, vom 15. Januar 1960: BArch, DK 107/7658.

41 Ministerrat der DDR, Staatssekretariat für Forschung und Technik, Beschlußprotokoll der konstituierenden Sitzung der „Kommission des Forschungsrates zur Ausarbeitung einer Grundkonzeption für die Behandlung industrieller Abprodukte“ am 17.02.1965, Berlin, den 23.02.1965, in: BArch, DK 107/8380.

42 Stubbe an Meusel, 17.02.1965: BArch, DK 107/8380.

43 Stubbe an den Direktor des Instituts für Landesforschung und Naturschutz Halle, Herrn Professor Dr. Bauer, 20.09.1965: BArch, DK 107/8380.

44 Prognosegruppe „Abprodukte und sozialistische Landeskultur“, Prognose Industrielle Abprodukte und planmäßige Gestaltung einer sozialistischen Landeskultur in der DDR, Berlin, September 1968: BArch DC 20-I/3/715, pag. 44-162.

45 Hauptaufgaben, Zielstellungen und Lösungswege zur Reinhaltung der Luft und der Gewässer im Bezirk Halle für den Prognosezeitraum beschlossen zur Bezirkstagssitzung am 23.05.1968, in: Mitteilungsblatt des Bezirkstages und Rat des Bezirkes Halle, Mai 1968, Nr. 2, 1-10: SHStA, 11430, Bezirkstag/Rat des Bezirkes Dresden, Nr. 38637. Zum „ökonomischen Experiment“ vgl. ausführlich Möller (2018: 201-208).

\section{Literatur}

Amberger, Alexander 2014. Bahro - Harich - Havemann. Marxistische Systemkritik und politisch Utopie in der DDR, Paderborn: Schöningh.

Ash, Mitchell G. 1995. Wissenschaftswandel in Zeiten politischer Umwälzungen: Entwicklungen, Verwicklungen, Abwicklungen. NTM. Internationale Zeitschrift für Geschichte und Ethik der Naturwissenschaften, Technik und Medizin (3): 1-21.

Ash, Mitchell G. 2001. Wissenschaft und Politik als Ressourcen für einander. Programmatische Überlegungen am Beispiel Deutschlands. In: Jürgen Büschenfeld, Heike Franz und Frank-Michael Kuhlemann (Hg.). Wissenschaftsgeschichte heute. Festschrift für Peter Lundgreen, Bielefeld: Verlag für Regionalgeschichte: 117-134.

Ash, Mitchell G. 2002. Wissenschaft und Politik als Ressourcen für einander. In: Rüdiger vom Bruch und Brigitte Kaderas (Hg.). Wissenschaften und Wissenschaftspolitik. Bestandsaufnahmen zu Formationen, Brüchen und Kontinuitäten im Deutschland des 20. Jahrhunderts, Stuttgart: Steine: 32-51.

Bauer, Ludwig, Hans Bohnstedt und Werner Schauer. Auch die Luft will gepflegt sein. NDBeilage Nr. 27 vom 4. Juli 1964: 5.

Bechmann Arnim (Hg.) 1991. Umweltpolitik in der DDR. Dokumente des Umbruchs, Berlin: Univ.-Bibliothek der TU.

Behrens, Hermann 2001. Die ersten Jahre - Naturschutz und Landschaftspflege in der SBZ/DDR von 1945 bis Anfang der 60er Jahre. In: Naturschutz in den Neuen Bundesländern - Ein Rückblick, hrsg. vom Institut für Umweltgeschichte und Regionalentwicklung e. V. (IUGR), 2. überarb. Aufl., Berlin: Verlag für Wissenschaft und Forschung: 15-86.

Behrens, Hermann 2005. Landschaftstage in der Deutschen Demokratischen Republik am Beispiel des Bezirks Neubrandenburg. In: Franz-Josef Brüggemeier und Jens Ivo Engels (Hg.). Natur- und Umweltschutz nach 1945. Konzepte, Konflikte, Kompetenzen, Frankfurt a.M.: Campus: $62-86$. 
Behrens, Hermann et al. 1993. Wurzeln der Umweltbewegung. Die „Gesellschaft für Natur und Umwelt" (GNU) im Kulturbund der DDR, Marburg: BdWI-Verlag.

Bergien, Rüdiger 2017. Im „Generalstab der Partei“. Organisationsstruktur und Herrschaftspraxis in der SED-Zentrale (1946-1989), Berlin: Akademie-Verlag.

Bessel, Richard und Ralph Jessen (Hg.) 1996. Die Grenzen der Diktatur. Staat und Gesellschaft in der DDR, Göttingen: Vandenhoeck und Ruprecht.

Büschenfeld, Jürgen 1997. Flüsse und Kloaken. Umweltfragen im Zeitalter der Industrialisierung (1870-1918), Stuttgart: Steiner.

Connelly, John 1997. Stalinistische Vielfalt: Hochschulpolitik im östlichen Mitteleuropa 1945-1955. In: Dieter Hoffmann und Kristie Macrakis (Hg.). Naturwissenschaft und Technik in der DDR, Berlin: Akademie Verlag: 89-104.

Deutscher Bundestag, 13. Wahlperiode. Schlußbericht der Enquete-Kommission „Überwindung der Folgen der SED-Diktatur im Prozeß der deutschen Einheit“, Drucksache 13/11000, 10.06.98.

Dieckmann, Johannes 1966. Sozialistische Landeskultur - eine nationale Aufgabe. Naturschutzarbeit in Berlin und Brandenburg (2): 67-68.

Eckhardt, Theodor 1953. Vom Naturschutz der Sowjetunion. Natur und Heimat (2): 137-143.

Engels, Jens Ivo 2006. Naturpolitik in der Bundesrepublik. Ideenwelt und politische Verhaltensstile in Naturschutz und Umweltbewegung 1950-1980, Paderborn: Schöningh.

Förtsch, Eckart 1997. Wissenschafts- und Technologiepolitik in der DDR. In: Dieter Hoffmann und Kristie Macrakis (Hg.). Naturwissenschaft und Technik in der DDR, Berlin: Akademie Verlag: 17-33.

Fulbrook, Mary 2008. Ein ganz normales Leben. Alltag und Gesellschaft in der DDR, Darmstadt: Wissenschaftliche Buchgesellschaft.

Fulbrook, Mary 2009. The Concept of „Normalisation“ and the GDR in Comparative Perspective. In: Mary Fulbrook (Hg.). Power and Society in the GDR, 1961-1979: The „Normalisation of Rule“?, New York: Berghahn: 1-30.

Fulbrook, Mary 2016. Questionable Concepts. Trust, Distrust and Normalisation. In: Mary Fulbrook. Erfahrung, Erinnerung, Geschichtsschreibung. Neue Perspektiven auf die deutschen Diktaturen, Göttingen: Wallstein: 62-86.

Gestwa, Klaus 2010. Die Stalinschen Großbauten des Kommunismus. Sowjetische Technikund Umweltgeschichte, 1948-1967, München: Oldenbourg.

Gilsenbach, Reimar 1953. Ohne Naturschutz keine Landeskultur. Natur und Heimat (2): 350.

Gilsenbach, Reimar 1959. Widerstreit von Wissenschaft und Praxis im Naturschutz? Natur und Heimat (8): 163-164.

Gilsenbach, Reimar 1961. Wohin gehst du, Naturschutz? 3. Teil. Natur und Heimat (10): 350-353.

Goetel, Walery 1959. Schutz den Naturreserven! Natur und Heimat (8): 164.

Gudermann, Rita 2006. „Natur nach Maß“. Hans Stubbe und das Verhältnis von Agrarwissenschaften und Umweltschutz in der DDR. In: Andreas Dix und Ernst Langthaler (Hg.). Grüne Revolutionen. Agrarsysteme und Umwelt im 19. und 20. Jahrhundert, Innsbruck: Studienverlag: 173-198.

Hasenöhrl, Ute 2011. Zivilgesellschaft und Protest. Eine Geschichte der Naturschutz- und Umweltbewegung in Bayern 1945-1980, Göttingen: Vandenhoeck und Ruprecht.

Heim, Susanne 2003. Kalorien, Kautschuk, Karrieren. Pflanzenzüchtung und landwirtschaftliche Forschung in Kaiser-Wilhelm-Instituten 1933-1945, Göttingen: Wallstein.

Hoffmann, Dierk 2013. Von Ulbricht zu Honecker. Die Geschichte der DDR 1949-1989, Berlin-Brandenburg: be.bra verlag.

Hossfeld, Uwe und Lennart Olsson 2002, From the Modern Synthesis to Lysenkoism, and Back? SCIENCE (297): 55-56.

Huff, Tobias 2015. Natur und Industrie im Sozialismus, Göttingen: Vandenhoeck und Ruprecht.

Hünemörder, Kai F. 2004. Die Frühgeschichte der globalen Umweltkrise und die Formierung der deutschen Umweltpolitik (1950-1973), Stuttgart: Steiner.

Jessen, Ralph 1998. Diktatorischer Elitewechsel und universitäre Milieus. Hochschullehrer in der SBZ/DDR (1945-1967). Geschichte und Gesellschaft. Zeitschrift für Historische Sozialwissenschaft (24): 24-54. 
Jessen, Ralph 2005. Between Control and Collaboration: The University in East Germany. In: John Connelly und Michael Grüttner (Hg.). Universities under Dictatorship, University Park, Pa.: Pennsylvania State University Press: $245-281$.

Käding, Edda 1999. Engagement und Verantwortung. Hans Stubbe, Genetiker und Züchtungsforscher. Eine Biographie, (=ZALF-Bericht Nr. 36), Müncheberg: Zentrum für Agrarlandschafts- und Landnutzungsforschung.

Kaiser, Monika 1997. Machtwechsel von Ulbricht zu Honecker. Funktionsmechanismen der SED-Diktatur in Konfliktsituationen 1962 bis 1972, Berlin: Akademie-Verlag.

Kalweit, Heino 1954. Die Verunreinigung der Gewässer in der Deutschen Demokratischen Republik. Deutsche Akademie der Landwirtschaftswissenschaften zu Berlin, Sitzungsberichte (3): 3-14.

Karlsch, Rainer 1993. Allein bezahlt? Die Reparationsleistungen der SBZ/DDR 1945-1953, Berlin: Ch.Links.

Kocka, Jürgen 1998. Wissenschaft und Politik in der DDR. In: Jürgen Kocka und Renate Mayntz, Wissenschaft und Wiedervereinigung: Disziplinen im Umbruch, Berlin: Akademie-Verlag: 435-459.

Kocka, Jürgen, Peter Nötzoldt und Peter Th. Walther 2002. Die Berliner Akademien der Wissenschaften 1945-1990. In: Jürgen Kocka (Hg.). Die Berliner Akademien der Wissenschaften im geteilten Deutschland 1945-1990, Berlin: Akademie Verlag: 363-457.

Köster, Roman 2017. Hausmüll. Abfall und Gesellschaft in Westdeutschland 1945-1990, Göttingen: Vandenhoeck \& Ruprecht.

Kowalczuk, Ilko-Sascha 1997. Legitimation eines neuen Staates: Parteiarbeiter an der historischen Front. Geschichtswissenschaft in der SBZ/DDR 1945 bis 1961, Berlin: Ch. Links.

Kuntsche, Siegfried 2014. Die Akademie der Landwirtschaftswissenschaften als Zweigakademie. In: Wolfgang Girnus und Klaus Meier (Hg.). Forschungsakademien in der DDR - Modelle und Wirklichkeit, Leipzig: Universitätsverlag: 335-379.

Kuntsche, Siegfried 2017. Die Akademie der Landwirtschaftswissenschaften 1951-1990, 2. Halbband, Leipzig: Universitätsverlag.

Laitko, Hubert 2009. Strategen, Organisatoren, Kritiker, Dissidenten - Verhaltensmuster prominenter Naturwissenschaftler der DDR in den 50er und 60er Jahren des 20. Jahrhunderts, Berlin: MPI für Wissenschaftsgeschichte. http://hdl.handle.net/11858/00001M-0000-002A-7EEC-1. Zugegriffen: 08.10.2018

Laitko, Hubert 2014. Forschungsakademien: Prämissen und Orientierungsfragen. Eröffnungsbeitrag auf der zweitägigen Tagung am 1. und 2. November 2012. In: Wolfgang Girnus und Klaus Meier (Hg.). Forschungsakademien in der DDR - Modelle und Wirklichkeit, Leipzig: Leipziger Universitätsverlag: 15-36.

Lindenberger, Thomas (Hg.) 1999. Herrschaft und Eigen-Sinn in der Diktatur. Studien zur Gesellschaftsgeschichte der DDR, Köln u. a.: Böhlau.

Lindenberger, Thomas 2016. Das Land der begrenzten Möglichkeiten. Machträume und Eigen-Sinn der DDR-Gesellschaft. Deutschland Archiv, 10.8.2016, Link: www.bpb.de/ 232099.

Lingner, Reinhold 1952. Landschaftsgestaltung, Berlin: Aufbau-Verlag.

Malycha, Andreas 2005. Wissenschaft und Politik in der DDR 1945 bis 1990. Ansätze zu einer Gesamtsicht. In: Clemens Burrichter und Gerald Diesener (Hg.). Reformzeiten und Wissenschaft. Beiträge zur DDR-Wissenschaftsgeschichte, Reihe B/Bd. 2, Leipzig: Universitätsverlag: 181-205.

Malycha, Andreas und Peter Jochen Winters 2009. Die Geschichte der SED. Von der Gründung bis zur Linkspartei, Bonn: Bundeszentrale für politische Bildung.

Meuschel, Sigrid 1992. Legitimation und Parteiherrschaft in der DDR. Zum Paradox von Stabilität und Revolution in der DDR 1945-1989, Frankfurt a.M.: Suhrkamp.

Meusel, Hermann 1953. Landeskultur und Naturschutz als nationale Aufgabe. Natur und Heimat (2): 323-326.

Meusel, Hermann 1958. Probleme der Landeskultur im Zeitalter der Technik. Berichte und Vorträge der Akademie der Landwirtschaftswissenschaften zu Berlin: Festsitzung und wissenschaftliche Tagung: 87-100.

Meusel, Hermann 1969. Forschung als Grundlage der Landeskultur. In: Deutscher Kulturbund (Hg.), Sozialistische Landeskultur. Referate und Diskussionsbeiträge der Eröff- 
nungsveranstaltung zur „Naturschutzwoche 1969“ und „Woche des Waldes" am 11. Mai 1969 in Zwickau, o.A.: 38-41.

Möller, Christian 2014. Der Traum vom ewigen Kreislauf? Abprodukte, Sekundärrohstoffe und Stoffkreisläufe im „Abfall-Regime“ der DDR (1945-1990). Technikgeschichte (81): 61-89.

Möller, Christian 2015. Zwischen Gestaltungseuphorie, Versagen und Ohnmacht: Umwelt, Staat und volkseigene Wirtschaft in der DDR. Zeitschrift für Unternehmensgeschichte (60): 141-167.

Möller, Christian 2018. Umweltpolitik in der DDR. Der umweltpolitische Aufbruch, die ökologische Krise und das Scheitern der „partizipatorischen Diktatur“, Dissertation zur Erlangung des Grades eines Doktors der Philosophie, Universität Bielefeld.

Musterle, Theodor 1963. Einflüsse des Wasservorkommens auf die langfristige Entwicklung von Wirtschaft und Leben. In: Ernst Rulo Welcker (Hg.). Das Wasser in seiner Bedeutung für Leben, Gesundheit und Krankheit des Menschen. Vorträge, gehalten auf der 4. Tagung der Medizinisch-Wissenschaftlichen Gesellschaft der DDR zum Studium der aktuellen Lebensbedingungen (13. Januar 1962 in Berlin), Jena: Gustav Fischer Verlag: 13-24.

Nesmejanow, A.N.1958. Naturschutz - ein Anliegen des ganzen Volkes. Mit Bildern aus kaukasischen Naturschutzgebieten von Herbert Görzig. Natur und Heimat (7): 136-138.

Nikolow, Sybilla und Arne Schirrmacher 2007. Das Verhältnis von Wissenschaft und Öffentlichkeit als Beziehungsgeschichte: Historiographische und systematische Perspektiven. In: Sybilla Nikolow und Arne Schirrmacher (Hg.). Wissenschaft und Öffentlichkeit als Ressourcen füreinander. Studien zur Wissenschaftsgeschichte im 20. Jahrhundert, Frankfurt a.M.: Campus: $11-36$.

Nikonow, Alexander Alexandrowitsch und Eberhard Schulze 2004. Drei Jahrhunderte Agrarwissenschaft in Russland: Von 1700 bis zur Gegenwart, Halle (Saale): Institut für Agrarentwicklung in Mittel- und Osteuropa.

Oberkrome, Willi 2005. Kontinuität und Wandel im deutschen Naturschutz 1930 bis 1970: Bemerkungen und Thesen. In: Franz-Josef Brüggemeier und Jens Ivo Engels (Hg.). Natur- und Umweltschutz nach 1945. Konzepte, Konflikte, Kompetenzen, Frankfurt a.M.: Campus: 23-37.

Obertreis, Julia 2012. Von der Naturbeherrschung zum Ökozid? Aktuelle Fragen einer Umweltzeitgeschichte Ost- und Ostmitteleuropas. Zeithistorische Forschungen/Studies in Contemporary History (9): 115-122.

Ortleb, Walter 1954. Technische Möglichkeiten der Abwasserreinigung. Deutsche Akademie der Landwirtschaftswissenschaften zu Berlin, Sitzungsberichte (3): 22-32.

Paucke, Horst 1994. Chancen für Umweltpolitik und Umweltforschung. Zur Situation in der ehemaligen DDR, Marburg: BdWI-Verlag.

Petschow, Ulrich, Jürgen Meyerhoff und Claus Thomasberger 1990. Umweltreport DDR. Bilanz der Zerstörung, Kosten der Sanierung, Strategien für den ökologischen Umbau, Frankfurt a.M.: S. Fischer.

Pniower, Georg 1953. Die Landwirtschaftlichen Produktionsgenossenschaften und die Aufgaben der Landeskultur. Natur und Heimat (2): 108-110.

Radkau, Joachim 2011. Die Ära der Ökologie. Eine Weltgeschichte, München: C.H. Beck.

Rochlitzer, Johann 1960. Über die gemeinsamen Aufgaben der Chemieindustrie und der Wasserwirtschaft im Siebenjahrplan. Chemische Technik (12): 627-635.

Roesler, Jörg 1990. Zwischen Plan und Markt. Die Wirtschaftsreform 1963-1970 in der DDR, Berlin: Haufe.

Roesler, Jörg 2006. System- oder konjunkturbedingte Unterschiede? Zur Umweltpolitik in der DDR und der Bundesrepublik in den 70er und 80er Jahren. Deutschland Archiv (39): 480-488.

Rösler, Marcus, Elisabeth Schwab, Markus Lambrecht (Hg.) 1990. Naturschutz in der DDR, Bonn: Economica-Verlag.

Sabrow, Martin 1999. Der Konkurs der Konsensdiktatur. Überlegungen zum inneren Zerfall der DDR aus kulturgeschichtlicher Perspektive. In: Konrad H. Jarausch und Martin Sabrow, Der Weg in den Untergang, Göttingen: Vandenhoeck \& Ruprecht: 83-116.

Sabrow, Martin 2001. Das Diktat des Konsenses. Geschichtswissenschaft in der DDR 1949-1969, München: Oldenbourg. 
Sabrow, Martin 2004. Zukunftspathos als Legitimationsressource. Zu Charakter und Wandel des Fortschrittsparadigmas in der DDR. In: Heinz-Gerhard Haupt und Jörg Requate (Hg.). Aufbruch in die Zukunft: Die 1960er Jahre zwischen Planungseuphorie und kulturellem Wandel. DDR, CSSR und Bundesrepublik im Vergleich, Weilerswist: Velbrück Wissenschaft: $165-184$.

Schöne, Jens 2010. Frühling auf dem Lande? Die Kollektivierung der DDR-Landwirtschaft. 3. durchgesehene Aufl. Berlin: Ch. Links.

Steiner, André 1999. Die DDR-Wirtschaftsreform der 60er Jahre. Konflikt zwischen Effizienzund Machtkalkül, Berlin: De Gruyter.

Stubbe, Michael 2002. Hans Stubbe - im Frieden für Wahrheit und Fortschritt - Engagement für Bewahrung und Nutzung von Naturressourcen. Beiträge zur Jagd-und Wildforschung (27): 79-124.

Tandler, Agnes Charlotte 2000. Geplante Zukunft. Wissenschaftler und Wissenschaftspolitik in der DDR 1955-1971, Freiberg: Technische Universität Bergakademie.

Titel, Werner 1969. Die Aufgaben der sozialistischen Landeskultur bei der Gestaltung des entwickelten Systems des Sozialismus in der DDR. In: Deutscher Kulturbund (Hg.). Sozialistische Landeskultur. Referate und Diskussionsbeiträge der Eröffnungsveranstaltung zur „Naturschutzwoche 1969“ und „Woche des Waldes" am 11. Mai 1969 in Zwickau, o.A.: 9-23.

Uekötter, Frank 2003. Von der Rauchplage zur ökologischen Revolution. Eine Geschichte der Luftverschmutzung in Deutschland und den USA 1880-1970, Essen: Klartext.

Verfassung der Deutschen Demokratischen Republik vom 6. April 1968, Berlin: Staatsverlag.

Wagner, Matthias 1992. Der Forschungsrat der DDR. Im Spannungsfeld von Sachkompetenz und Ideologieanspruch. 1954-April 1962, Dissertation zur Erlangung des akademischen Grades doctor oeconomiae, Humboldt-Universität zu Berlin.

Weber, Hermann 2000. Die DDR 1945-1990, 3. überarb. u. erw. Aufl., München: Oldenbourg.

Weinitschke, Hugo 1963. Naturschutzprobleme vor der Volkskammer. Naturschutzarbeit und naturkundliche Forschung in Sachsen (5): 33-35.

Weinitschke, Hugo 1969. 15 Jahre Naturschutzgesetz in der DDR. In: Deutscher Kulturbund (Hg.). Sozialistische Landeskultur. Referate und Diskussionsbeiträge der Eröffnungsveranstaltung zur "Naturschutzwoche 1969“ und „Woche des Waldes“ am 11. Mai 1969 in Zwickau, o.A.: 23-29.

Weinitschke, Hugo 1980. Naturschutz gestern, heute, morgen, Leipzig: Urania-Verl.

Weißhaupt, Fritz 1959. Widerstreit von Wissenschaft und Praxis im Naturschutz. Erwiderung auf den Artikel von R. Gilsenbach. Natur und Heimat (8): 323-325.

Wensierski, Peter 1986. Von oben nach unten wächst gar nichts. Umweltzerstörung und Protest in der DDR, Frankfurt a.M.: Fischer.

Wiedemann, Kurt 1958a. Landschaftsschutz für die Sächsische Schweiz. Pläne für den gemeinsamen Nationalpark Böhmische und Sächsische Schweiz. Natur und Heimat (7): $152-155$.

Wiedemann, Kurt 1958b. Landschaftsschutz für die Sächsische Schweiz. Fortsetzung und Schluß. Natur und Heimat (7): 176-178.

Wöbse, Anna-Katharina 2012. Weltnaturschutz. Umweltdiplomatie in Völkerbund und Vereinten Nationen 1920-1950, Frankfurt a.M.: Campus.

Wolle, Stefan 1999. Die heile Welt der Diktatur. Alltag und Herrschaft in der DDR 1971-1989, 2. durchges. Aufl., Bonn: Bundeszentrale für politische Bildung.

Wolle, Stefan 2011. Aufbruch nach Utopia. Alltag und Herrschaft in der DDR 1961-1971, Bonn: Bundeszentrale für politische Bildung.

Wolschke-Bulmahn, Joachim und Peter Fibich 2004. Vom Sonnenrund zur Beispiellandschaft. Entwicklungslinien in Deutschland, dargestellt am Werk von Georg Pniower (1896-1960), Hannover: Institut für Grünplanung und Gartenarchitektur.

Würth, Gerhard 1985. Umweltschutz und Umweltzerstörung in der DDR, Frankfurt a.M.: Peter Lang.

Zeller, Thomas 2003. „Ganz Deutschland sein Garten“: Alwin Seifert und die Landschaft des Nationalsozialismus. In: Joachim Radkau und Frank Uekötter (Hg.). Naturschutz und Nationalsozialismus, Frankfurt a.M.: Campus: 273-307. 
Christian Möller

Professur für Wirtschafts- und Sozialgeschichte

Heinrich-Heine-Universität Düsseldorf

Universitätsstraße 1

40225 Düsseldorf

Deutschland

Christian.Moeller@uni-duesseldorf.de 